\title{
3 Research Suare \\ FKBP51 Modulates Hippocampal Size and Function in Post-translational Regulation of Parkin
}

\section{Bin Qiu}

Yale University

\section{Zhaohui Zhong}

Peking University People's Hospital

\section{Shawn Righter}

Indiana University Purdue University at Indianapolis

\section{Yuxue Xu}

ILAS: Chinese Academy of Medical Sciences Institute of Laboratory Animal Sciences

Jun Wang

ILAS: Chinese Academy of Medical Sciences Institute of Laboratory Animal Sciences

\section{Ran Deng}

ILAS: Chinese Academy of Medical Sciences Institute of Laboratory Animal Sciences

\section{Chao Wang}

ILAS: Chinese Academy of Medical Sciences Institute of Laboratory Animal Sciences

\section{Kent E Williams}

Indiana University School of Medicine

\section{Yao-Ying Ma}

Indiana University School of Medicine

\section{Gavriil Tsechpenakis}

Indiana University Purdue University at Indianapolis

\section{Tiebing Liang}

Indiana University School of Medicine https://orcid.org/0000-0002-8445-3765

\section{Weidong Yong ( $\nabla$ wyong@iu.edu )}

Indiana University School of Medicine

\section{Research Article}

Keywords: Fkbp51, hippocampus, neuron, Parkin, Artificial Intelligence

Posted Date: May 19th, 2021

DOI: https://doi.org/10.21203/rs.3.rs-493867/v1 
License: (c) (i) This work is licensed under a Creative Commons Attribution 4.0 International License. Read Full License

Version of Record: A version of this preprint was published at Cellular and Molecular Life Sciences on March 1st, 2022. See the published version at https://doi.org/10.1007/s00018-022-04167-8. 


\section{Abstract}

FK506-binding protein 51 (encoded by Fkpb51) has been associated with stress-related mental illness. To identify its function, we studied the morphological consequences of Fkbp51 deletion. Artificial Intelligence-assist morphological analysis identified that Fkbp51 knock-out (KO) mice possess more elongated CA and DG but shorter in height in coronal section when compared to WT. Primary cultured Fkbp51 KO hippocampal neurons were shown to exhibit larger dendritic outgrowth than wild-type (WT) controls, pharmacological manipulation experiments suggest that this may occur through regulation of microtubule-associated protein. Both in vitro primary culture and in vivo labeling support that FKBP51 regulates microtubule-associated protein expression. Furthermore, in the absence of differences in mRNA expression, Fkbp51 KO hippocampus exhibited decreases in Blll-tubulin, MAP2, and Tau protein levels, but a greater than 2.5-fold increase in Parkin protein. Overexpression and knock-down FKBP51 demonstrated that FKBP51 negatively regulates Parkin in a dose-dependent and ubiquitin-mediated manner. These results indicate a potential novel post-translational regulatory of Parkin by FKBP51 and significance of their interaction on disease onset.

\section{Introduction}

FK506-binding protein 51 (FKBP51, encoded by Fkbp5) belongs to a subclass of immunophilin proteins and has peptidyl-prolyl cis-trans isomerase (PPlase) activity that is crucial for protein folding (Schiene and Fischer, 2000). One of the well-studied functions of FKBP51 is its role as a co-chaperone of heat shock protein 90 (Hsp90) in the formation of the glucocorticoid receptor (GR) complex, which is a central contributor to the stress response that modifies the pathophysiology of stress-induced conditions (Schmidt et al., 2015; Szymanska et al., 2009; Tatro et al., 2010). For example, Fkbp51 has been associated with depression, post-traumatic stress disorder (PTSD), and other psychiatric disorders (Fani et al., 2013; Wang et al., 2018; Xie et al., 2010). Newly emerging data indicate that FKBP51 may also play an important role in neuronal development, neurological diseases, and as a potential target for disease treatment (Gaali et al., 2015; Lieberman et al., 2016; Matosin et al., 2018; O'Leary et al., 2011; Schmidt et al., 2012; Wagner et al., 2012). Interestingly, interactions with childhood adversity and emotion processing indicate that FKBP51 may play a role in neuronal plasticity through epigenetic regulation or unknown function (Arlt et al., 2013; Binder et al., 2008; Gerritsen et al., 2017; Lekman et al., 2008).

The hippocampus is critical for learning and memory and experiences continuous neurogenesis into adulthood, and its volume has a relatively high level of heritability (Kremen et al., 2010; Ming and Song, 2005; Renteria et al., 2014). Hippocampal volume can be affected by neurogenesis or dendritic atrophy, which can be influenced by genetic predisposition, disease conditions, and certain therapies (Hibar et al., 2017; Horgusluoglu-Moloch et al., 2019; Levy-Gigi et al., 2013; Levy-Gigi et al., 2015). Ample evidence supports the association between hippocampal volume and neurological and psychiatric diseases (Onoue et al., 2013; Smith, 2005; Thompson et al., 2004). Importantly, FKBP51 has been associated with hippocampal volume alterations in PTSD patients (Dannlowski et al., 2015; Levy-Gigi et al., 2013; Yun et al., 2020). The hippocampus is rich in corticosteroid receptors as well as FKBP51, and stress-induced 
glucocorticoid elevations result in many morphological and molecular changes in the hippocampus (Conrad et al., 2017; Qiu et al., 2016). However, no direct evidence from knocking out Fkbp51 to support its role in hippocampal morphology and potential mechanism in disease development.

In this study, we examined the hippocampal morphology differences between $F k b p 51$ knock-out (KO) and wild-type (WT) mice using Artificial Intelligence (Al) approach to determine the effects of Fkbp51 ablation on neuronal development. Pharmacological manipulations were applied to understand the role of $F k b p 51$ in microtubule dynamics. We measured the mRNA and protein levels of MAP2, Tubulin, Tau, and Parkin in Fkbp51 KO and WT mice, and the functional relationships, particularly between FKBP51 and Parkin, were thoroughly investigated by knocking down and overexpressing Fkbp51. Co-immunoprecipitation (Co-IP) and co-transfection experiments support the role of FKBP51 in the regulation of Parkin. These findings suggest that $F k b p 51$ plays a critical role in the dendritic complexity of neurons, and is reflected by differences of deep cell layers of CA and DG between KO and WT in vivo, highlighting a potential mechanism underpinning morphological, synaptic, and molecular function differences.

\section{Results}

\section{Fkbp51 gene deletion leads to altered hippocampal size}

FKBP51 has been linked to hippocampal volume in human studies (Levy-Gigi et al., 2013). To directly confirm such an association, we examined if any changes in hippocampus morphology occur using the Fkbp51 KO mouse model. Because the use of individuals to define the morphological changes could be biased and limited, artificial intelligence (Al) tools were developed and applied to perform unbiased and in-depth surveys of the hippocampal area, using distinguishable cell layers, such as neuron cell layers of cornu ammonis (CA) and the dentate gyrus (DG) as landmarks. Brain positions were registered and calibrated by application of $\mathrm{Al}$-assist data analysis (Figure 1A), and application of methods previously established by ourselves and others (Rother, 2004; Tsechpenakis and Chatzis, 2011). In brief, each image was preprocessed in grayscale with the locally adaptive histogram equalization method (Zuiderveld, 1994), followed by contrast enhancement and hybrid probability-driven deformable modeling (Tsechpenakis and Chatzis, 2011). The Al analysis suggested alterations in hippocampal size and DG size (Figure 1). Figure 1B depicts an overview of the results. The yellow region in the image is the segmentation outcome, while red lines are the resulting smooth centerlines along the interface, also shown in magnified views. Eight measurements were calculated for both left and right sides. The bar plots indicate corresponding measurements from all examined stacks across all depths. The results are shown in the numbered plots corresponding to the annotated measurements in the displayed image. The cortex plus corpus callosum (CC) lengths were not significantly different between WT and KO. However, the height of hippocampus was found to be shorter in KO than WT, the length of the CA was found to be longer in $\mathrm{KO}$ than WT. The curve radii at CA1 and CA3 were found to be larger in KO than WT indicating more flattened curves. Similarly, longer DG lengths, shorter DG heights, and smaller DG curve radii were observed in KO relative to WT. Interestingly, genotype-specific left and right sides differences were found (Figure 2B); and hippocampal height, CA1 curve radius, and DG length are KO-specific, CA3 curve radius 
and DG height have opposite trend between KO and WT. These data demonstrate that CA are DG of KO mice are significantly more elongated (horizontally) compared to WT, while the height of the hippocampus in $\mathrm{KO}$ is shorter (vertically). Our data demonstrate that the morphological consequences of knocking out $F k b p 51$, an intrinsic gene regulatory mechanism during development.

\section{Fkbp51 ablation affects neuronal development}

A previous study demonstrated that Fkbp51 plays a role in neurite outgrowth using cell lines and primary cultured hippocampal neurons from rats (Quinta et al., 2010), but this role has not been directly studied using KO animal models. To uncover the effects of Fkbp51 deletion on neuronal development and to identify its specificity, we examined the morphology of primary hippocampal neurons cultured from Fkbp51 KO and WT mice. The neurons were IF-labeled with Blll-tubulin, MAP2, and DAPI. At 3 days in vitro (DIV3), axonal specification and primary dendritic outgrowth had begun (Figure 2). The distribution patterns of MAP2 and BIII-tubulin were similar between WT and Fkbp51 KO neurons, but Fkbp51 KO neurons exhibited lower MAP2 signal intensities (Figure 2, DIV3 panel). At DIV5 one long axonal outgrowth and few dendritic branches were clearly present in WT neurons. In Fkbp51 KO neurons, one shorter axonal outgrowth and multiple overgrown dendritic branches were observed, an effect not evident in WT neurons (Figure 2. DIV5 panel). Another striking difference was that KO cells had only weak labeling around the somatic and nuclear membranes, but WT cells had higher signal intensity for both BIII-tubulin and MAP2 labelingin these areas (Figure 2, DIV5, magnified insets). Neuronal morphology differences were initially observed at DIV3, but became more evident at DIV5 and DIV9 (Figure 2). More representative neurons with different target labeling are shown in the Supplemental (S) materials. Triple labeling of Blll-tubulin, F-actin, and DAPI (S-Figure 1A) clearly shows more dendrite growth in KO. Consistently, BIII-tubulin, Tau (labeling axon), and DAPI IF labeling (S- Figure 1B) confirmed the differences between WT and Fkbp51 KO neurons with similar phenotypes as described above. These results suggest that knocking out $F k b p 51$ has a significant impact on neuronal development, and microtubule-associated protein expression levels are affected. The result is consistent with the role microtubule and actin dynamics play in determining neuronal polarization (Yogev et al., 2016).

\section{Microtubule-stabilizing drug altered hippocampal neuron development in Fkbp51 KO mice}

Neurite outgrowth is essential for wiring the nervous system during development and in certain disease conditions (Barnes and Polleux, 2009). We examined the involvement of FKBP51 in neuron development through the regulation of microtubules. Pharmacological interventions were applied to further investigate the function of FKBP51 in neurite outgrowth and its effect on microtubule dynamics during neuron development. In this experiment, a microtubule-stabilizing agent (Taxol; $3 \mathrm{nM}$ ) (Letourneau and Ressler, 1984) or a microtubule-destabilizing agent (nocodazole; $50 \mathrm{nM}$ ) (Chang et al., 2008) was applied to Fkbp51 KO and WT primary hippocampal neuron cultures. Similar to above observations, in untreated conditions, KO hippocampal neurite overgrowth was evident with $\beta$ III-tubulin and MAP2 labeling. As before, one long axon with a few short neurites (arrow head) were observed in WT neurons (Figure 3A- 
untreated panel). Following treatment with Taxol, the MAP2 compartment was affected in WT neurons (Figure 3A, Taxol- WT panel), likely due to the ability of Taxol to promote microtubule stability and enhance protrusion of polymerizing dynamic microtubules to the growth cone. In Fkbp51 KO neurons, Taxol treatment increased MAP2 labeling in the soma and neurites and reduced neurite numbers to the level of WT neurons (Figure 3B). While nocodazole treatment disturbed the normal dynamic distribution of MAP, we observed neurite retraction in both $\mathrm{KO}$ and WT (Figure 3A-nocodazole panel) neurons, which was more obvious and possibly associated with delayed neurite growth, in KO. The average number of primary neurites from soma was counted from multiple neurons per genotype under these treatment conditions (Figure 3B). The observation that pharmacological treatment can normalize dendrite overgrowth present in $F k b p 51 \mathrm{KO}$ neurons suggests that the neuronal phenotype of the $F k b p 51 \mathrm{KO}$ is a result of alterations in microtubule-related proteins.

\section{Fkbp51 elimination affects Tubulin, MAP2, and Tau protein expression}

Cytoskeletal proteins, including microtubules, neurofilaments, and actin microfilaments, are important for maintaining neuronal morphology and function (Gomez and Letourneau, 2014). MAPs, particularly MAP2, Tau, and tubulin, are critical for neuron development and function. Given that in vitro dendrite outgrowth was apparently affected by knocking out $F k b p 51$, we examined the in vivo expression levels of MAP mRNA and proteins in the hippocampi of Fkbp51 KO and WT mice. First, using quantitative real-time PCR, the levels of mRNA expression of Tubb3, Map2, and Tau were determined. Unexpectedly, similar levels of mRNA expression were found between Fkbp51 KO and WT mice (Figure 4A). We then examined protein expression using $\beta$ III-tubulin, MAP2, and Tau IF labeling, immunohistochemical (IHC) labeling, and Western blotting. In DG, CA1, and CA3 subfields, significantly different expression patterns were found KO and WT mice when observe in higher magnification. In the DG, the granular cell layer (GCL), hilus region $(\mathrm{HR})$, and molecular layer (ML) showed lower levels of MAP2 staining in the KO than WT (Figure 4B, DG panel). Notably, the deep layer (yellow arrow pointed) of the GCL exhibited less intense labeling of MAP2 in Fkbp51 KO mice than in WT mice and result in no visible labeling in this subfield. Similarly, low expression of MAP2 was observed in stratum oriens (Ori), pyramidal (Pyr) neuron layer, and stratum radiatum (Rad) of Fkbp51 KO compared to WT; a pattern evident in the deep layer cell of the Pyr neuron layer of the CA1 and CA3 subregions (Figure 4B). The differences in MAP2 expression in hippocampal deep layers and superfical layers indicate that neuronal development has been affected in vivo in KO mice (Figure 4B, hand drawing model) .

BIII-tubulin and MAP2 were co-labeled and measured by IF as well. Lower levels of MAP2 expression were consistently observed in the DG and CA1 of KO mice, relative to those in WT mice (S-figure 2, MAP2 panel). For $\beta$ III-tubulin, lower levels were also evident in the hilar region (HR), GCL, and molecular layers (ML) of the DG. Merged images displayed the labeling differences in the outer layers between KO and WT. The pyramidal (Pyr) neurons at CA1 possessed significantly lower signal, evidence of deep cell layer differences in Fkbp51 KO mice compared to WT mice. Particularly, greatly reduced labeling in the Rad layer was observed in the CA1 region of $F k b p 51$ KO, signifying that FKBP51 affects tubulin in Pyr neurons. Furthermore, IF labeling of BlII-tubulin in the CA1 Rad layer of Fkbp51 KO clearly demonstrated 
reduced expression, more pronounced in the alveus (Alv) and lacunosum-molecular (LM) layers (S-Figure 2).

The extension of the neuronal dendritic tree into the inner and medial/outer molecular layers (IML and $\mathrm{M} / \mathrm{OML}$ ) of the $\mathrm{DG}$ is associated with the stress response and disease onset (Dioli et al., 2019; Seifan et al., 2015). The expression of MAPs was further examined using IHC to gain cellular resolution. For MAP2 labeling, KO mice showed less intensity than WT mice within the Pyr neuron cell layer, as well as in the apical branch in the Rad subregion, which is consistent with what was observed in the cultured neurons

(Figure 4C-MAP2 panel). Similarly, Tau had lower expression in the CA1 in KO mice, compared to WT mice (Figure 4C, Tau panel). IF labeling further confirmed these observations and revealed lower Tau expression, particularly in the Alv of CA1 (S-Figure 2 Tau panel). Both BIII-tubulin and MAP2 IHC labeling were consistent with IF labeling results, but more prominent in terms of cellular details. $\beta$ III-tubulin showed more clear sublayer differences in Rad by IF labeling. Western blotting was used to quantify the total protein expression in the hippocampus and to confirm the low expression of these proteins including phosphorylated MAP2 in KO (Figure 4D-E). Consistent with our in vitro primary neuron culture findings, in vivo $\beta$ III-tubulin, MAP2, and Tau labeling indicated clear differences in protein levels as well as sublayer expression differences in hippocampus between Fkbp51 KO and WT mice.

\section{Increased Parkin levels occur in Fkbp51 KO mice via post-translational regulation}

Given the importance of Parkin in microtubule stabilization, we compared the expression of Parkin between Fkbp51 KO and WT mice and assessed possible mechanisms of FKBP51 interplay with Parkin that could affect neuronal morphology. Strikingly, IHC labeling indicated clear differences in Parkin expression in hippocampal sublayers between Fkbp51 KO and WT mice (Figure 5A). The Pyr neurons at CA1 possessed significantly higher levels of Parkin in Fkbp51 KO mice than in WT mice, with a stronger intensity of labeling in the Rad region. Similarly, higher expression of Parkin was observed in the GCL and $\mathrm{HR}$ in the DG of $F k b p 51 \mathrm{KO}$ mice than in WT mice (Figure 5A). Consistent with the IHC labeling results, the Parkin protein was found to be significantly elevated in the Fkbp51 KO hippocampus $(p<0.001)$ compared to WT hippocampus (Figure 5B), although the lack of a difference in mRNA expression (Figure 5C) suggests that Parkin could be post-translationally regulated by FKBP51. These data suggest that hippocampal Parkin is expressed more abundantly in Fkbp51 KO mice than in WT mice.

\section{FKBP51 affects Parkin expression and stability and is involved in Parkin ubiquitin-mediated degradation}

The effect of Fkbp51 knockdown on Parkin expression in vitro was performed in an attempt to experimentally test the relationship between Parkin and FKBP51. Utilizing the human neuroblastoma cell line SH-SY5Y, we studied the effect of siRNA knockdown of Fkbp51 on Parkin expression levels and cellular morphology. Compared to the scramble siRNA control, Fkbp51 siRNA treatment reduced the IF signal intensity of MAP2 and Tau. Additionally, we observed that a higher intensity of F-actin labeling shifted from soma to dendrites when comparing the Fkbp51 siRNA group and control, with a significant increase in the Parkin signal in perinuclear area. Importantly, the Fkbp51 siRNA treatment group also exhibited altered morphology, with an increase from two to many neurite outgrowths, a result consistent 
with Fkbp51 KO (Figure 6A). Western blotting analysis confirmed a reduction in FKBP51 and an increase in Parkin levels in Fkbp51 siRNA-treated SH-SY5Y cells compared to control cells (Figure 6B). Thus, Fkbp51 siRNA altered microtubule-associated protein and F-actin expression.

As an E3 ubiquitin ligase, Parkin mediates the targeting of protein degradation machinery. Proper regulation of Parkin is critical, either via degradation or transitioning between active and inactive conformations (Chaugule and Walden, 2016). We performed in vitro overexpression experiments to test whether FKBP51 regulates Parkin expression. The Flag-Fkbp51 plasmid was transfected into cells. Following FKBP51 overexpression, endogenous Parkin expression was dramatically decreased in a dosedependent manner (Figure 6C). This finding suggests that FKBP51 expression reduces Parkin expression, potentially by affecting Parkin stability. To investigate this possibility, cells were transfected with FlagFkbp51 prior to the addition of cycloheximide (CHX), a protein synthesis inhibitor. The cells were harvested at 2, 4, 12, and $24 \mathrm{hr}$ post-CHX treatment, and Parkin protein levels were examined. Following $\mathrm{CHX}$ treatment, Parkin protein levels declined steadily in Flag-Fkbp51-treated cells (Figure 6D), suggesting that FKBP51 directly diminishes Parkin stability.

To study the role of ubiquitin in this process, His-Parkin, Flag-Fkbp51, and HA-ubiquitin were cotransfected in different combinations. As shown in Figure 6E, compared to His-Parkin expression alone (Figure 6E, lane 1), co-transfection of both Parkin and ubiquitin (HA-UB) resulted in diminished Parkin levels (Figure 6E, lane 2). Intriguingly, decreased endogenous FKBP51 expression was also observed (Figure 6E, lane 2). The data indicate ubiquitin-mediated Parkin and FKBP51 degradation. As expected, co-transfection of FKBP51 and His-Parkin without ubiquitin resulted in a significant decrease in Parkin expression relative to that of cells transfected with His-Parkin alone (Figure 6E, lane 3 vs lane 1) demonstrating that overexpression of FKBP51 reduces Parkin. Co-transfection of ubiquitin with FKBP51 and Parkin dramatically reduced endogenous FKBP51, Flag-FKBP51, and Parkin expression (Figure 6E, lane 4), suggesting that ubiquitin enhances FKBP51 activity. The bottom panel is the full gel blot for HAUB. Taken together, these results indicate that FKBP51 regulates Parkin expression in a dose- and ubiquitin-mediated manner. Next, the direct interaction between the Parkin and FKBP51 proteins was investigated using co-IP.

It has been reported that FKBP51 can interact with neuronal MAPs, Tau, and Tubulin (Chambraud et al., 2007; Matosin et al., 2018), but the possibility that FKBP51 interacts directly with Parkin has not been previously investigated. To further explore the interactions among FKBP51, Parkin, Blll-tubulin, and Tau, co-IP experiments were performed in SH-SY5Y cells. As shown in Figure 6F, FKBP51 precipitated with $\beta$ IIItubulin and Tau, confirming previous findings. FKBP51 also precipitated with Parkin, demonstrating that a probable interaction. Furthermore, evidence was found that suggests Parkin precipitates with MAP2 (Figure 6F), supporting previous research that indicated the possible association of these two proteins (Ren et al., 2003). We conclude that FKBP51 interacts with each of these proteins, but whether directly or indirectly requires further determination.

\section{Discussion}


Herein we report that Fkbp51 impacts neuronal morphology and regulates Parkin expression. We demonstrated alterations of hippocampus size by measuring hippocampal and DG dimensions in Fkbp51 KO and WT mice. Application of Al tools significantly advanced our understanding of these morphological changes in a more detailed fashion, and allowed us to identify the differences between KO and WT and between hemispheres. We reported that microtubule-associated protein changes in Fkbp51 $\mathrm{KO}$ mice may be implicated in dendritic outgrowth. Pharmacological manipulations supported that Fkbp51 regulates microtubule dynamics. Further investigation found that deletion of $F k b p 51$ resulted in no obvious alteration of mRNA expression but significant changes in several protein levels, including decreases in Blll-tubulin, MAP2, and Tau, while Parkin levels were more than doubled. We focused our investigation on FKBP51 and Parkin and provided a mechanistic understanding of the FKBP51 and Parkin interaction by overexpression or siRNA knockdown of $F k b p 51$, which revealed an inverse relationship between Fkbp51 and Parkin. Co-transfection experiments identified a dose-dependent and ubiquitin-mediated negative correlation between FKBP51 and Parkin. Moreover, co-IP experiments confirmed that FKBP51 binds Blll-tubulin, Tau, and Parkin. Our discovery of the role of Fkbp51 in neuronal development via regulation of Parkin is novel and its role in the regulation of MAP2 and tubulin levels during neuronal polarization may be the key for advancing future research on their associations with disease onset.

There are known genetic determinants of hippocampus volume and its association with mental illness (Ashbrook et al., 2014; Hibar et al., 2017; Na et al., 2018; Pujol et al., 2020; Zhang et al., 2019). Previous literature demonstrates that FKBP51 is critical for neuronal development and stress-related psychiatric diseases (Lieberman et al., 2016; Matosin et al., 2018). For example, Fkbp51 has been associated with depression, PTSD, and other psychiatric disorders, and PTSD patients display hippocampal volume changes (Fani et al., 2013; Wang et al., 2018; Xie et al., 2010). Thus, our KO model represents a genetically relevant in vivo model for identifying the long-term effects of neurological and morphological alterations. Hippocampal CA and DG volumes are smaller in MDD patients, however exposure to early life stress results in increased volume, and more interestingly, the FKBP51 genotype is associated with different responses to treatment of those patients with early life stress. Additionally, FKBP51 haplotype was shown to modulate the resting brain activity of parents who lost their only child (Levy-Gigi et al., 2013; Mikolas et al., 2019; Qi et al., 2020). These data suggest that hippocampal volume is at least partially determined by FKBP51 genetics. Distinct genotypes respond differently to treatment, indicating a role for FKBP51 in gene-environmental interaction. Furthermore, FKBP51 does play a role in neuronal plasticity and brain activity (Fani et al., 2016). Indeed, we also observe alterations in synaptic plasticity in Fkbp51 KO mice (Qiu et al., 2019). The breadth of these profound findings suggests an extensive role for FKBP51 in normal biological function and in disease onset. However, a full mechanistic understanding of its modes of action in humans is missing critical details.

FKBP51 genotype-dependent increases in coupling between the left amygdala and left hippocampus have been shown to suggest its role in emotional processing and differences between hemispheres (Holz et al., 2015). Another study found a positive correlation between the severity of repression and left hippocampal volume in a subgroup of PTSD with a specific FKBP51 genotype (Yun et al., 2020). 
Interestingly, glucocorticoid receptor (GR), a protein that forms a complex with FKBP51 to carry out its function, has a "protective" genotype that responds to emotional trauma and affects the reductions in left hippocampal volumes (Malhi et al., 2019). In our study, the Al tool enabled a more accurate quantification of morphological changes in the hippocampus. In addition to the genotype-specific differences between left and right hemispheres was quite interesting. Thus, our Fkbp51 KO model also supports the notion that genetic predisposition of FKBP51 in hippocampal morphology and hemisphere differences.

The hippocampus plays key roles in spatial navigation and episodic memory, and segments along the transverse axis of the hippocampal pyramidal cell layer reflect their morphological and functional differences (Eichenbaum, 2000; Slomianka et al., 2011). Previous research identified these layers may possess differences in circuit function and activity, particularly differences between deep and superficial neurons correlated with physiological functions and connections to basket cells (Slomianka et al., 2011; Soltesz and Losonczy, 2018). In our study, we have identified a role of FKBP51 in neuronal development via the post-translational regulation of microtubule associated protein expression (i.e., down regulation of MAP2, but upregulation of Parkin). MAP2 labeling in the cultured neurons and in vivo hippocampal labeling indicated that MAP2 expression levels in neurons are consistent. Particularly, striking were MAP2 labeling differences in the deep cell layer of GCL of DG, as well as deep pyramidal neurons of CA1 and CA3. Clearly, Fkbp51 KO produces changes in neuronal development and hippocampal morphology.

Microtubules are essential for neuronal development and interact with microtubule-associated proteins (MAPs), Tau, and Parkin (Ren et al., 2003). Mutations and variations in these proteins are associated with major neurodevelopmental and neurodegenerative diseases, such as Parkinson's Disease (PD) and Alzheimer's disease (AD) (Buee et al., 2000; Corti et al., 2011; Goate et al., 1991; Matsumoto et al., 2010; Takahashi et al., 2008). Recent research has established that Parkin, a multifunctional ubiquitin ligase, binds and stabilizes microtubules, regulates gene expression, and participates in mitochondrial homeostasis (Darios et al., 2003; Ren et al., 2003; Williams et al., 2015; Yang et al., 2005). Parkin can also directly form Tubulin dimers via ubiquitination and through interactions with hallmark proteins of $A D$ (Olah et al., 2011; Ren et al., 2003). Parkin absence accelerates microtubule aging in dopaminergic neurons (Cartelli et al., 2018; Ren et al., 2009). Interestingly, FKBP51 binds Tubulin and associates with MAPs, Tau, Hsp90, and other chaperones to guide neuronal differentiation (Chambraud et al., 2007; Quinta et al., 2010). However, no knowledge about the relationship between FKBP51 and Parkin.

In this study, we revealed the potential interaction of these two genes. Intuitively, if the morphological alterations that were observed in vitro persist in the whole organism, they must affect the entire neuronal organization in vivo. Greater dendrite outgrowth in the Fkbp51 KO could alter normal neuronal function, resulting in connectivity differences. Our group and others recently found $F k b p 51$ plays a role in synaptic plasticity (Blair et al., 2019; Qiu et al., 2019). The neuronal polarization is a dynamic process including microtubule protein transportation, cross-linking between microtubules and other proteins, as well as the internal traction force in the axon (Courchet et al., 2013; Wilson and Gonzalez-Billault, 2015; Xu et al., 2013; Yadaw et al., 2019). In Fkbp51 KO neurons, the microtubule dynamic was altered due to downregulation of microtubule-associated protein, but significantly up-regulation of Parkin, which stabilized 
the microtubules. Parkin and FKBP51 interaction may play a prominent role in the regulation of microtubule-related proteins, such as MAP2, Tubulin, and Tau. It is probable that FKBP51 performs these functions via its isomerase activity and its involvement in the phosphorylation, ubiquitination, and lipidation of other proteins (Rein, 2020a; Rein, 2020b). We propose a model for their interaction based on our findings (Figure 7). Normally, FKBP51 interacts with Parkin and inhibit Parkin activity, allowing microtubule-related proteins (the substrates Tubulin, Map2, Tau, etc.) to maintain in normal degradation. In the absence of FKBP51, their interactions can result in enhanced Parkin activity, enhanced substrate degradation. We speculate that these changes produce a new homeostasis of the cytoskeletal network and function, which can affect neuronal development and hippocampal formation, resulting in alterations in plasticity and connectivity (Fani et al., 2016; Quinta et al., 2010). These changes would further explain the contribution of FKBP51 in stress-related mental illnesses (Binder et al., 2008; Xing et al., 2015; Zannas et al., 2019).

The discovery that Parkin activity is regulated by FKBP51 opens a new strategy for the treatment of Parkin-associated neurological diseases. It is known that some forms of PD exhibit inactivated Parkin activity (LaVoie et al., 2005), and it has been suggested that chronic stress conferred by the metabolism of dopamine in dopaminergic neurons may render those neurons more dependent on Parkin (EkholmReed et al., 2013). We observed ubiquitin-mediated degradation of Parkin, which is consistent with a previous report that Parkin self-regulation could be exacerbated via ubiquitin-assisted degradation (Chew et al., 2011). Evidence of FKBP51-dependent and ubiquitin-mediated regulation of Parkin expression, and doubled Parkin protein in the hippocampus of $F k b p 51 \mathrm{KO}$ mice suggested an upstream regulatory role for FKBP51 in the control of Parkin activity. Parkin has been associated with multiple diseases and functions as an E3 ligase in the ubiquitination pathway that controls protein quality and gene expression (Darios et al., 2003; Ren et al., 2003; Williams et al., 2015; Yang et al., 2005). Both FKBP51 and Parkin are involved in neuronal function. Additionally, FKBP51 and Parkin share several overlapping functions related to disease development (Corti et al., 2011; Kim et al., 2006). FKBP51 is implicated in stress-related disorders, including PTSD, depression, and addiction (Binder et al., 2008; Huang et al., 2014; Lekman et al., 2008; Qiu et al., 2016), and a recent meta-analysis of GWAS found PARK2 is an additional gene associated with PTSD (Nievergelt et al., 2019). Thus, the molecular level interaction and the regulation of the neuronal cytoskeleton are highly possible.

In summary, this research identified a novel function of FKBP51 as a determinant of neuronal development and hippocampal morphology. The mechanism may be related to its role in the posttranslational regulation of microtubule-related protein expression, through which it directly influences Parkin function and activity. These findings provide a foundation for further studies on the role of FKBP51 in neuronal function and disease development and may aid in understanding the value of FKBP51 as a genetic factor for depression, PTSD, and other mental illnesses. Revealing these novel functions of FKBP51 may lead to the development of rational pharmacological interventions.

\section{Materials And Methods}




\section{Animals}

All experimental protocols were reviewed and approved by the Animal Care and Research Advisory Committee of the Institute of Laboratory Animal Sciences at the Chinese Academy of Medical Sciences and the Indiana University School of Medicine. The animals were maintained in facilities fully accredited by the Association for the Assessment and Accreditation of Laboratory Animal Care (AAALAC). The development of Fkbp51 KO mice was described in a previous publication (Yong et al., 2007).

\section{H\&E staining and measurement of hippocampal morphology}

Brain sections from adult $F k b p 51 \mathrm{KO}$ and WT animals were analyzed for anatomical differences. Coronal brain sections from a total of 6 male mice ( 3 Fkbp51 KO mice and 3 WT controls) at 8 weeks of age were obtained. The whole brains were fixed in $4 \%$ paraformaldehyde, embedded in paraffin, and serially sectioned at a thickness of $4 \mu \mathrm{m}$. Sections from both $3 \mathrm{KO}(\mathrm{N}=82$ sections) and $3 \mathrm{WT}$ ( $\mathrm{N}=82$ sections) mice near Bregma $-2.18 \mathrm{~mm}$ were collected for image analysis. H\&E staining was applied as previously described (Yong et al., 2007). The architecture of the hippocampus was observed on sections corresponding to the same anatomical plane using a light microscope and camera (Leica CTR6000 with DFC450 C, Wetzlar, Germany).

\section{Al-assisted morphological analysis}

Machine learning was applied to accurately assess hippocampal morphology. A total of 164 coronal view images from 6 stacks of 3 WT and 3 Fkbp51-KO mouse brains were analyzed ( 2 stacks containing 30 images and 1 stack containing 22 images from each genotype). Each image in a stack was processed separately. The 2D hippocampus interface was first segmented at each stack depth, and the centerline of the segmented regions was approximated to obtain two curves, one for CA and one for DG. Based on the local characteristics (position and curvature) of each curve, contextual landmarks of interest were located, upon which 2D shape features describing the hippocampus were calculated. To tackle the spatially varying contrast and color/intensity statistics throughout the images, a local, adaptive approach was followed. Each image was preprocessed in grayscale with the locally adaptive histogram equalization method for contrast enhancement, as previously described (Zuiderveld, 1994). The hybrid probability-driven deformable model was applied as established prior (Tsechpenakis and Chatzis, 2011). For initial training of the region classification component of the method, $\sim 200$ random samples (small regions) of the interface of interest and another $\sim 200$ samples from the surrounding regions, from all ( 30) images of four stacks (two WT and two KO) were manually obtained. For each examined image, after the initial probability field was obtained, the evolution of the deformable region produced more samples for the positive and negative hypotheses (desired interface and surroundings, respectively). The embedded classifier was thus continually re-trained and the image probability field was updated throughout the model evolution, incorporating local image statistics using the published method (Tsechpenakis and Chatzis, 2011). The final segmentation results did show some inaccuracies, primarily at image regions with a high degree of intensity ambiguity. It is worth noting that the semi-automatic 
graph cuts were also tested with acceptable results, but also with a lot of manual region annotation (Rother, 2004).

\section{Primary hippocampal neuron culture and immunofluorescence}

Primary cultures of hippocampal neurons were prepared from the hippocampi of mouse embryos at embryonic day 15.5 (E15.5) as previously described, with some modifications (Qiu et al., 2013). For the drug treatment groups, Taxol (Millipore, Billerica, MA, USA) or nocodazole (Millipore, Billerica, MA, USA) was added to culture medium at final concentrations of $3 \mathrm{nM}$ or $50 \mathrm{nM}$, respectively. DMSO was applied to the untreated group as a vehicle control. Neuronal development was assessed using IF labeling of $\beta$ IIItubulin, MAP2, and DAPI. The IF of primary cultured hippocampal neurons on day 3, 5, or 9 in vitro (DIV 3 , 5 , or 9) was evaluated for MAP2, Blll-tubulin, and DAPI, as previously described (Wang et al., 2014). All experiments were repeated independently 3 times. The antibodies used in this study are listed in Supplementary Table S1.

\section{Western blotting analysis and quantitative real-time PCR}

Proteins were isolated using radioimmunoprecipitation assay (RIPA) lysis buffer and total mRNA was isolated in TRIzol $\circledast$ from hippocampi (Beyotime, Jiangsu, China) $(\mathrm{N}=3-5)$. Western blotting was performed as previously described (Qiu et al., 2013). The antibodies used are listed in Supplementary Table S1. Reverse transcription (RT) and quantitative real-time PCR were conducted as previously described (Gu et al., 2016). The relative mRNA expression levels were normalized to $R p / 7$, which was not differentially expressed between the Fkbp $51 \mathrm{KO}$ and WT groups. The primers utilized are listed in Supplementary Table S2. All experiments were repeated independently 3-5 times.

\section{Co-IP and Parkin stability}

The human neuroblastoma cell lines SH-SY5Y and HEK293 were cultured with DMEM containing 10\% FBS, $1 \%$ penicillin and streptomycin, and $2 \mathrm{mM}$ GlutaMax in a humidified $37^{\circ} \mathrm{C}$ incubator with $5 \% \mathrm{CO}_{2}$. The cells were transfected with the Flag-Fkbp51 (HG11487-CF, Sino Biological, Beijing, China), His-Park2 (HG12092-NH, Sino Biological, Beijing, China) or HA-ubiquitin (\#18712, Addgene, Cambridge, MA, USA) plasmid for overexpression or with the human Fkbp51 siRNA (sc-35380, Santa Cruz Biotech, Inc., Dallas, TX, USA) for knock-down, using Lipofectamine 3000 (L3000008, Life Technologies, Gaithersburg, MD, USA) according to the manufacturer's recommendations. Thirty-six hours after transfection, the cells were used for co-IP experiments. A co-IP kit (\#26149, Life Technologies, Gaithersburg, MD, USA) was utilized according to the manufacturer's instructions. All experiments were repeated independently 3-5 times. The antibodies used in this study are listed in Supplementary Table S1.

For the Parkin stability study, SH-SY5Y cells were transfected with Flag-Fkbp51 or the control plasmid and treated with $\mathrm{CHX}(2 \mu \mathrm{g} / \mathrm{ml})$ to inhibit further protein synthesis after $12 \mathrm{hr}$ of transfection. The cells were harvested in RIPA buffer 15, 30, 45, or 60 min after CHX treatment. Aliquots of $100 \mu \mathrm{g}$ of total protein were analyzed by Western blotting. 


\section{Statistical analysis}

All values are presented as the mean \pm the standard error of the mean (SEM). Student's t-test was used for comparisons between two groups, while two-way analysis of variance (ANOVA) was performed to compare multiple group differences, followed by a Student-Newman-Keuls test for multiple comparisons. GraphPad Prism was used for data analysis (GraphPad Software Inc., San Diego, CA, USA), and significance was determined as $p<0.05$.

\section{Declarations}

\section{Ethics approval and consent to participate}

Animal studies has been reviewed and approved by the Animal Care and Research Advisory Committee of the Institute of Laboratory Animal Sciences at the Chinese Academy of Medical Sciences and the Indiana University School of Medicine. No humans were included in this study.

\section{Consent for publication}

All authors reviewed and agreed to publish the current finding.

\section{Availability of data and material}

All data will be available for the public after publication. No large data set needs to be deposit to the public repository.

\section{Competing interests}

No competing interests

\section{Funding}

This research was supported by grants from CAMS Innovation Fund for Medical Sciences (CIFMS) (2017I2M-3-015), the National Science Foundation of China (No. 81700751 and 2013CB945001).

\section{Conflict of Interest}

All authors have no conflicts of interest to declare.

\section{Author Contribution}

Authors contributed to the study conception and design: [Weidong Yong], [Tiebing Liang], [Yao-ying Ma], and [Gavriil Tsechpenakis]. Material preparation, data collection and analysis were performed by [Bin Qiu], [Zhaohui Zhong], [Shawn Righter], [Yuxue Xu], [Jun Wang], [Ran Deng], [Chao Wang], [Kent E. Williams] and [Gavriil Tsechpenakis]. Data curation and formal analysis: [Bin Qiu], [Zhaohui Zhong], [Shawn Righter], and [Kent William], Investigation: All authors participated. Al assisted data analysis 
Methodology: [Gavriil Tsechpenakis] and [Shawn Righter]. The first draft of the manuscript was written by [Bin Qiu] [Tiebing Liang], [Weidong Yong] and all authors commented on previous versions of the manuscript. All authors read and approved the final manuscript. Funding acquisition: [Weidong Yong].

\section{Acknowledgments}

We would also like to express our appreciation to Indiana Alcohol Research Center and Dr. Weinian Shou for the support in initiating this long-term project.

\section{* Authors' information}

Bin Qiu: kennyqiu@live.com

Zhaohui Zhong: zhongzhaohui@pkuph.edu.cn

Shawn Righter: gtsechpe@indiana.edu

Yuxue Xu: xuyuxue1127@126.com

Jun Wang: junwang138@sina.com

Ran Deng: 598046836@qq.com

Chao Wang:1358560339@qq.com

Kent E. Williams : kew5@iu.edu

Yao-ying Ma :ym9@iu.edu

Gavriil Tsechpenakis: gtsechpe@indiana.edu

Tiebing Liang: tliang@iu.edu

Weidong Yong: yongwd@iu.edu

\section{References}

1. Arlt, S., C. Demiralay, B. Tharun, O. Geisel, N. Storm, M. Eichenlaub, J.T. Lehmbeck, K. Wiedemann, B. Leuenberger, and H. Jahn. 2013. Genetic risk factors for depression in Alzheimer's disease patients. Curr Alzheimer Res. 10:72-81.

2. Ashbrook, D.G., R.W. Williams, L. Lu, J.L. Stein, D.P. Hibar, T.E. Nichols, S.E. Medland, P.M. Thompson, and R. Hager. 2014. Joint genetic analysis of hippocampal size in mouse and human identifies a novel gene linked to neurodegenerative disease. BMC Genomics. 15:850.

3. Barnes, A.P., and F. Polleux. 2009. Establishment of axon-dendrite polarity in developing neurons. Annu Rev Neurosci. 32:347-381. 
4. Binder, E.B., R.G. Bradley, W. Liu, M.P. Epstein, T.C. Deveau, K.B. Mercer, Y. Tang, C.F. Gillespie, C.M. Heim, C.B. Nemeroff, A.C. Schwartz, J.F. Cubells, and K.J. Ressler. 2008. Association of FKBP5 polymorphisms and childhood abuse with risk of posttraumatic stress disorder symptoms in adults. JAMA. 299:1291-1305.

5. Blair, L.J., M. Criado-Marrero, D. Zheng, X. Wang, S. Kamath, B.A. Nordhues, E.J. Weeber, and C.A. Dickey. 2019. The Disease-Associated Chaperone FKBP51 Impairs Cognitive Function by Accelerating AMPA Receptor Recycling. eNeuro. 6.

6. Buee, L., T. Bussiere, V. Buee-Scherrer, A. Delacourte, and P.R. Hof. 2000. Tau protein isoforms, phosphorylation and role in neurodegenerative disorders. Brain Res Brain Res Rev. 33:95-130.

7. Cartelli, D., A. Amadeo, A.M. Calogero, F.V.M. Casagrande, C. De Gregorio, M. Gioria, N. Kuzumaki, I. Costa, J. Sassone, A. Ciammola, N. Hattori, H. Okano, S. Goldwurm, L. Roybon, G. Pezzoli, and G. Cappelletti. 2018. Parkin absence accelerates microtubule aging in dopaminergic neurons. Neurobiol Aging. 61:66-74.

8. Chambraud, B., H. Belabes, V. Fontaine-Lenoir, A. Fellous, and E.E. Baulieu. 2007. The immunophilin FKBP52 specifically binds to tubulin and prevents microtubule formation. FASEB J. 21:2787-2797.

9. Chang, Y.C., P. Nalbant, J. Birkenfeld, Z.F. Chang, and G.M. Bokoch. 2008. GEF-H1 couples nocodazole-induced microtubule disassembly to cell contractility via RhoA. Mol Biol Cell. 19:21472153.

10. Chaugule, V.K., and H. Walden. 2016. Specificity and disease in the ubiquitin system. Biochem Soc Trans. 44:212-227.

11. Chew, K.C., N. Matsuda, K. Saisho, G.G. Lim, C. Chai, H.M. Tan, K. Tanaka, and K.L. Lim. 2011. Parkin mediates apparent E2-independent monoubiquitination in vitro and contains an intrinsic activity that catalyzes polyubiquitination. PloS one. 6:e19720.

12. Conrad, C.D., J.B. Ortiz, and J.M. Judd. 2017. Chronic stress and hippocampal dendritic complexity: Methodological and functional considerations. Physiol Behav. 178:66-81.

13. Corti, O., S. Lesage, and A. Brice. 2011. What genetics tells us about the causes and mechanisms of Parkinson's disease. Physiological reviews. 91:1161-1218.

14. Courchet, J., T.L. Lewis, Jr., S. Lee, V. Courchet, D.Y. Liou, S. Aizawa, and F. Polleux. 2013. Terminal axon branching is regulated by the LKB1-NUAK1 kinase pathway via presynaptic mitochondrial capture. Cell. 153:1510-1525.

15. Dannlowski, U., H.J. Grabe, K. Wittfeld, J. Klaus, C. Konrad, D. Grotegerd, R. Redlich, T. Suslow, N. Opel, P. Ohrmann, J. Bauer, P. Zwanzger, I. Laeger, C. Hohoff, V. Arolt, W. Heindel, M. Deppe, K. Domschke, K. Hegenscheid, H. Volzke, D. Stacey, H. Meyer Zu Schwabedissen, H. Kugel, and B.T. Baune. 2015. Multimodal imaging of a tescalcin (TESC)-regulating polymorphism (rs7294919)specific effects on hippocampal gray matter structure. Mol Psychiatry. 20:398-404.

16. Darios, F., O. Corti, C.B. Lucking, C. Hampe, M.P. Muriel, N. Abbas, W.J. Gu, E.C. Hirsch, T. Rooney, M. Ruberg, and A. Brice. 2003. Parkin prevents mitochondrial swelling and cytochrome c release in mitochondria-dependent cell death. Hum Mol Genet. 12:517-526. 
17. Dioli, C., P. Patricio, N. Sousa, N. Kokras, C. Dalla, S. Guerreiro, M.A. Santos-Silva, A.C. Rego, L. Pinto, E. Ferreiro, and I. Sotiropoulos. 2019. Chronic stress triggers divergent dendritic alterations in immature neurons of the adult hippocampus, depending on their ultimate terminal fields. Trans/ Psychiatry. 9:143.

18. Eichenbaum, H. 2000. A cortical-hippocampal system for declarative memory. Nat Rev Neurosci. 1:41-50.

19. Ekholm-Reed, S., M.S. Goldberg, M.G. Schlossmacher, and S.I. Reed. 2013. Parkin-dependent degradation of the F-box protein Fbw7beta promotes neuronal survival in response to oxidative stress by stabilizing Mcl-1. Mol Cell Biol. 33:3627-3643.

20. Fani, N., D. Gutman, E.B. Tone, L. Almli, K.B. Mercer, J. Davis, E. Glover, T. Jovanovic, B. Bradley, I.D. Dinov, A. Zamanyan, A.W. Toga, E.B. Binder, and K.J. Ressler. 2013. FKBP5 and attention bias for threat: associations with hippocampal function and shape. JAMA Psychiatry. 70:392-400.

21. Fani, N., T.Z. King, J. Shin, A. Srivastava, R.C. Brewster, T. Jovanovic, B. Bradley, and K.J. Ressler. 2016. Structural and Functional Connectivity in Posttraumatic Stress Disorder: Associations with Fkbp5. Depress Anxiety. 33:300-307.

22. Gaali, S., A. Kirschner, S. Cuboni, J. Hartmann, C. Kozany, G. Balsevich, C. Namendorf, P. FernandezVizarra, C. Sippel, A.S. Zannas, R. Draenert, E.B. Binder, O.F. Almeida, G. Ruhter, M. Uhr, M.V. Schmidt, C. Touma, A. Bracher, and F. Hausch. 2015. Selective inhibitors of the FK506-binding protein 51 by induced fit. Nat Chem Biol. 11:33-37.

23. Gerritsen, L., Y. Milaneschi, C.H. Vinkers, A.M. van Hemert, L. van Velzen, L. Schmaal, and B.W. Penninx. 2017. HPA Axis Genes, and Their Interaction with Childhood Maltreatment, are Related to Cortisol Levels and Stress-Related Phenotypes. Neuropsychopharmacology. 42:2446-2455.

24. Goate, A., M.C. Chartier-Harlin, M. Mullan, J. Brown, F. Crawford, L. Fidani, L. Giuffra, A. Haynes, N. Irving, L. James, and et al. 1991. Segregation of a missense mutation in the amyloid precursor protein gene with familial Alzheimer's disease. Nature. 349:704-706.

25. Gomez, T.M., and P.C. Letourneau. 2014. Actin dynamics in growth cone motility and navigation. $J$ Neurochem. 129:221-234.

26. Gu, H., Y. Cao, B. Qiu, Z. Zhou, R. Deng, Z. Chen, R. Li, X. Li, Q. Wei, X. Xia, and W. Yong. 2016. Establishment and phenotypic analysis of an Mstn knockout rat. Biochemical and biophysical research communications. 477:115-122.

27. Hibar, D.P., H.H.H. Adams, N. Jahanshad, G. Chauhan, J.L. Stein, E. Hofer, M.E. Renteria, J.C. Bis, A. Arias-Vasquez, M.K. Ikram, S. Desrivieres, M.W. Vernooij, L. Abramovic, S. Alhusaini, N. Amin, M. Andersson, K. Arfanakis, B.S. Aribisala, N.J. Armstrong, L. Athanasiu, T. Axelsson, A.H. Beecham, A. Beiser, M. Bernard, S.H. Blanton, M.M. Bohlken, M.P. Boks, J. Bralten, A.M. Brickman, O. Carmichael, M.M. Chakravarty, Q. Chen, C.R.K. Ching, V. Chouraki, G. Cuellar-Partida, F. Crivello, A. Den Braber, N.T. Doan, S. Ehrlich, S. Giddaluru, A.L. Goldman, R.F. Gottesman, O. Grimm, M.E. Griswold, T. Guadalupe, B.A. Gutman, J. Hass, U.K. Haukvik, D. Hoehn, A.J. Holmes, M. Hoogman, D. Janowitz, T. Jia, K.N. Jorgensen, N. Karbalai, D. Kasperaviciute, S. Kim, M. Klein, B. Kraemer, P.H. Lee, D.C.M. Liewald, L.M. 
Lopez, M. Luciano, C. Macare, A.F. Marquand, M. Matarin, K.A. Mather, M. Mattheisen, D.R. McKay, Y. Milaneschi, S. Munoz Maniega, K. Nho, A.C. Nugent, P. Nyquist, L.M.O. Loohuis, J. Oosterlaan, M. Papmeyer, L. Pirpamer, B. Putz, A. Ramasamy, J.S. Richards, S.L. Risacher, R. Roiz-Santianez, N. Rommelse, S. Ropele, E.J. Rose, N.A. Royle, T. Rundek, P.G. Samann, A. Saremi, C.L. Satizabal, L. Schmaal, A.J. Schork, L. Shen, J. Shin, E. Shumskaya, A.V. Smith, E. Sprooten, L.T. Strike, A. Teumer, et al. 2017. Novel genetic loci associated with hippocampal volume. Nat Commun. 8:13624.

28. Holz, N.E., A.F. Buchmann, R. Boecker, D. Blomeyer, S. Baumeister, I. Wolf, M. Rietschel, S.H. Witt, M.M. Plichta, A. Meyer-Lindenberg, T. Banaschewski, D. Brandeis, and M. Laucht. 2015. Role of FKBP5 in emotion processing: results on amygdala activity, connectivity and volume. Brain Struct Funct. 220:1355-1368.

29. Horgusluoglu-Moloch, E., S.L. Risacher, P.K. Crane, D. Hibar, P.M. Thompson, A.J. Saykin, K. Nho, and I. Alzheimer's Disease Neuroimaging. 2019. Genome-wide association analysis of hippocampal volume identifies enrichment of neurogenesis-related pathways. Sci Rep. 9:14498.

30. Huang, M.C., M.L. Schwandt, J.A. Chester, A.M. Kirchhoff, C.F. Kao, T. Liang, J.D. Tapocik, V.A. Ramchandani, D.T. George, C.A. Hodgkinson, D. Goldman, and M. Heilig. 2014. FKBP5 moderates alcohol withdrawal severity: human genetic association and functional validation in knockout mice. Neuropsychopharmacology. 39:2029-2038.

31. Kim, J.M., K.H. Lee, Y.J. Jeon, J.H. Oh, S.Y. Jeong, I.S. Song, J.M. Kim, D.S. Lee, and N.S. Kim. 2006. Identification of genes related to Parkinson's disease using expressed sequence tags. DNA Res. 13:275-286.

32. Kremen, W.S., E. Prom-Wormley, M.S. Panizzon, L.T. Eyler, B. Fischl, M.C. Neale, C.E. Franz, M.J. Lyons, J. Pacheco, M.E. Perry, A. Stevens, J.E. Schmitt, M.D. Grant, L.J. Seidman, H.W. Thermenos, M.T. Tsuang, S.A. Eisen, A.M. Dale, and C. Fennema-Notestine. 2010. Genetic and environmental influences on the size of specific brain regions in midlife: the VETSA MRI study. Neuroimage. 49:1213-1223.

33. LaVoie, M.J., B.L. Ostaszewski, A. Weihofen, M.G. Schlossmacher, and D.J. Selkoe. 2005. Dopamine covalently modifies and functionally inactivates parkin. Nat Med. 11:1214-1221.

34. Lekman, M., G. Laje, D. Charney, A.J. Rush, A.F. Wilson, A.J. Sorant, R. Lipsky, S.R. Wisniewski, H. Manji, F.J. McMahon, and S. Paddock. 2008. The FKBP5-gene in depression and treatment response-an association study in the Sequenced Treatment Alternatives to Relieve Depression (STAR*D) Cohort. Biol Psychiatry. 63:1103-1110.

35. Letourneau, P.C., and A.H. Ressler. 1984. Inhibition of neurite initiation and growth by taxol. J Cell Biol. 98:1355-1362.

36. Levy-Gigi, E., C. Szabo, O. Kelemen, and S. Keri. 2013. Association among clinical response, hippocampal volume, and FKBP5 gene expression in individuals with posttraumatic stress disorder receiving cognitive behavioral therapy. Biol Psychiatry. 74:793-800.

37. Levy-Gigi, E., C. Szabo, G. Richter-Levin, and S. Keri. 2015. Reduced hippocampal volume is associated with overgeneralization of negative context in individuals with PTSD. Neuropsychology. 
29:151-161.

38. Lieberman, R., S. Armeli, D.M. Scott, H.R. Kranzler, H. Tennen, and J. Covault. 2016. FKBP5 genotype interacts with early life trauma to predict heavy drinking in college students. Am J Med Genet B Neuropsychiatr Genet. 171:879-887.

39. Malhi, G.S., P. Das, T. Outhred, C. Dobson-Stone, L. Irwin, D. Gessler, R. Bryant, and Z. Mannie. 2019. Effect of stress gene-by-environment interactions on hippocampal volumes and cortisol secretion in adolescent girls. Aust N Z J Psychiatry. 53:316-325.

40. Matosin, N., T. Halldorsdottir, and E.B. Binder. 2018. Understanding the Molecular Mechanisms Underpinning Gene by Environment Interactions in Psychiatric Disorders: The FKBP5 Model. Biol Psychiatry. 83:821-830.

41. Matsumoto, L., H. Takuma, A. Tamaoka, H. Kurisaki, H. Date, S. Tsuji, and A. Iwata. 2010. CpG demethylation enhances alpha-synuclein expression and affects the pathogenesis of Parkinson's disease. PLoS One. 5:e15522.

42. Mikolas, P., L. Tozzi, K. Doolin, C. Farrell, V. O'Keane, and T. Frodl. 2019. Effects of early life adversity and FKBP5 genotype on hippocampal subfields volume in major depression. J Affect Disord. 252:152-159.

43. Ming, G.L., and H. Song. 2005. Adult neurogenesis in the mammalian central nervous system. Annu Rev Neurosci. 28:223-250.

44. Na, K.S., E. Won, J. Kang, A. Kim, S. Choi, Y.K. Kim, M.S. Lee, and B.J. Ham. 2018. Interaction effects of oxytocin receptor gene polymorphism and depression on hippocampal volume. Psychiatry Res Neuroimaging. 282:18-23.

45. Nievergelt, C.M., A.X. Maihofer, T. Klengel, E.G. Atkinson, C.Y. Chen, K.W. Choi, J.R.I. Coleman, S. Dalvie, L.E. Duncan, J. Gelernter, D.F. Levey, M.W. Logue, R. Polimanti, A.C. Provost, A. Ratanatharathorn, M.B. Stein, K. Torres, A.E. Aiello, L.M. Almli, A.B. Amstadter, S.B. Andersen, O.A. Andreassen, P.A. Arbisi, A.E. Ashley-Koch, S.B. Austin, E. Avdibegovic, D. Babic, M. Baekvad-Hansen, D.G. Baker, J.C. Beckham, L.J. Bierut, J.I. Bisson, M.P. Boks, E.A. Bolger, A.D. Borglum, B. Bradley, M. Brashear, G. Breen, R.A. Bryant, A.C. Bustamante, J. Bybjerg-Grauholm, J.R. Calabrese, J.M. Caldasde-Almeida, A.M. Dale, M.J. Daly, N.P. Daskalakis, J. Deckert, D.L. Delahanty, M.F. Dennis, S.G. Disner, K. Domschke, A. Dzubur-Kulenovic, C.R. Erbes, A. Evans, L.A. Farrer, N.C. Feeny, J.D. Flory, D. Forbes, C.E. Franz, S. Galea, M.E. Garrett, B. Gelaye, E. Geuze, C. Gillespie, A.G. Uka, S.D. Gordon, G. Guffanti, R. Hammamieh, S. Harnal, M.A. Hauser, A.C. Heath, S.M.J. Hemmings, D.M. Hougaard, M. Jakovljevic, M. Jett, E.O. Johnson, I. Jones, T. Jovanovic, X.J. Qin, A.G. Junglen, K.I. Karstoft, M.L. Kaufman, R.C. Kessler, A. Khan, N.A. Kimbrel, A.P. King, N. Koen, H.R. Kranzler, W.S. Kremen, B.R. Lawford, L.A.M. Lebois, C.E. Lewis, S.D. Linnstaedt, A. Lori, B. Lugonja, J.J. Luykx, M.J. Lyons, J. Maples-Keller, C. Marmar, A.R. Martin, et al. 2019. International meta-analysis of PTSD genome-wide association studies identifies sex- and ancestry-specific genetic risk loci. Nat Commun. 10:4558.

46. O'Leary, J.C., 3rd, S. Dharia, L.J. Blair, S. Brady, A.G. Johnson, M. Peters, J. Cheung-Flynn, M.B. Cox, G. de Erausquin, E.J. Weeber, U.K. Jinwal, and C.A. Dickey. 2011. A new anti-depressive strategy for the 
elderly: ablation of FKBP5/FKBP51. PLoS One. 6:e24840.

47. Olah, J., O. Vincze, D. Virok, D. Simon, Z. Bozso, N. Tokesi, I. Horvath, E. Hlavanda, J. Kovacs, A. Magyar, M. Szucs, F. Orosz, B. Penke, and J. Ovadi. 2011. Interactions of pathological hallmark proteins: tubulin polymerization promoting protein/p25, beta-amyloid, and alpha-synuclein. J Biol Chem. 286:34088-34100.

48. Onoue, T., H. Toda, and Y. Nakai. 2013. [Childhood stress and depression]. Nihon Shinkei Seishin Yakurigaku Zasshi. 33:105-110.

49. Pujol, N., A. Mane, D. Berge, G. Mezquida, S. Amoretti, L. Perez, A. Gonzalez-Pinto, F. Barcones, M.J. Cuesta, G. Sanchez-Tomico, E. Vieta, J. Castro-Fornieles, M. Bernardo, M. Parellada, and P.E. GROUP. 2020. Influence of BDNF and MTHFR polymorphisms on hippocampal volume in first-episode psychosis. Schizophr Res. 223:345-352.

50. Qi, R., Y. Luo, L. Zhang, Y. Weng, W. Surento, N. Jahanshad, Q. Xu, Y. Yin, L. Li, Z. Cao, P.M. Thompson, and G.M. Lu. 2020. FKBP5 haplotypes and PTSD modulate the resting-state brain activity in Han Chinese adults who lost their only child. Trans/ Psychiatry. 10:91.

51. Qiu, B., S. Hu, L. Liu, M. Chen, L. Wang, X. Zeng, and S. Zhu. 2013. CART attenuates endoplasmic reticulum stress response induced by cerebral ischemia and reperfusion through upregulating BDNF synthesis and secretion. Biochem Biophys Res Commun. 436:655-659.

52. Qiu, B., S.E. Luczak, T.L. Wall, A.M. Kirchhoff, Y. Xu, M.Y. Eng, R.B. Stewart, W. Shou, S.L. Boehm, J.A. Chester, W. Yong, and T. Liang. 2016. The FKBP5 Gene Affects Alcohol Drinking in Knockout Mice and Is Implicated in Alcohol Drinking in Humans. Int J Mol Sci. 17.

53. Qiu, B., Y. Xu, J. Wang, M. Liu, L. Dou, R. Deng, C. Wang, K.E. Williams, R.B. Stewart, Z. Xie, W. Ren, Z. Zhao, W. Shou, T. Liang, and W. Yong. 2019. Loss of FKBP5 Affects Neuron Synaptic Plasticity: An Electrophysiology Insight. Neuroscience. 402:23-36.

54. Quinta, H.R., D. Maschi, C. Gomez-Sanchez, G. Piwien-Pilipuk, and M.D. Galigniana. 2010. Subcellular rearrangement of hsp90-binding immunophilins accompanies neuronal differentiation and neurite outgrowth. J Neurochem. 115:716-734.

55. Rein, T. 2020a. Peptidylprolylisomerases, Protein Folders, or Scaffolders? The Example of FKBP51 and FKBP52. Bioessays:e1900250.

56. Rein, T. 2020b. Post-translational modifications and stress adaptation: the paradigm of FKBP51. Biochem Soc Trans. 48:441-449.

57. Ren, Y., H. Jiang, F. Yang, K. Nakaso, and J. Feng. 2009. Parkin protects dopaminergic neurons against microtubule-depolymerizing toxins by attenuating microtubule-associated protein kinase activation. J Biol Chem. 284:4009-4017.

58. Ren, Y., J. Zhao, and J. Feng. 2003. Parkin binds to alpha/beta tubulin and increases their ubiquitination and degradation. J Neurosci. 23:3316-3324.

59. Renteria, M.E., N.K. Hansell, L.T. Strike, K.L. McMahon, G.I. de Zubicaray, I.B. Hickie, P.M. Thompson, N.G. Martin, S.E. Medland, and M.J. Wright. 2014. Genetic architecture of subcortical brain regions: common and region-specific genetic contributions. Genes Brain Behav. 13:821-830. 
60. Rother, C., Kolmogorov, V., and Blake, A. . 2004. GrabCut - Interactive Foreground Extraction using Iterated Graph Cuts. . ACM Transactions on Graphics (SIGGRAPH). 23:309-314.

61. Schiene, C., and G. Fischer. 2000. Enzymes that catalyse the restructuring of proteins. Curr Opin Struct Biol. 10:40-45.

62. Schmidt, M.V., M. Paez-Pereda, F. Holsboer, and F. Hausch. 2012. The prospect of FKBP51 as a drug target. ChemMedChem. 7:1351-1359.

63. Schmidt, U., D.R. Buell, I.A. Ionescu, N.C. Gassen, F. Holsboer, M.B. Cox, B. Novak, C. Huber, J. Hartmann, M.V. Schmidt, C. Touma, T. Rein, and L. Herrmann. 2015. A role for synapsin in FKBP51 modulation of stress responsiveness: Convergent evidence from animal and human studies. Psychoneuroendocrinology. 52:43-58.

64. Seifan, A., K.S. Marder, J. Mez, J.M. Noble, E.P. Cortes, J.P. Vonsattel, and L.S. Honig. 2015. Hippocampal laminar distribution of tau relates to Alzheimer's disease and age of onset. $J$ Alzheimers Dis. 43:315-324.

65. Slomianka, L., I. Amrein, I. Knuesel, J.C. Sorensen, and D.P. Wolfer. 2011. Hippocampal pyramidal cells: the reemergence of cortical lamination. Brain Struct Funct. 216:301-317.

66. Smith, M.E. 2005. Bilateral hippocampal volume reduction in adults with post-traumatic stress disorder: a meta-analysis of structural MRI studies. Hippocampus. 15:798-807.

67. Soltesz, I., and A. Losonczy. 2018. CA1 pyramidal cell diversity enabling parallel information processing in the hippocampus. Nat Neurosci. 21:484-493.

68. Szymanska, M., B. Budziszewska, L. Jaworska-Feil, A. Basta-Kaim, M. Kubera, M. Leskiewicz, M. Regulska, and W. Lason. 2009. The effect of antidepressant drugs on the HPA axis activity, glucocorticoid receptor level and FKBP51 concentration in prenatally stressed rats. Psychoneuroendocrinology. 34:822-832.

69. Takahashi, K., C. Uchida, R.W. Shin, K. Shimazaki, and T. Uchida. 2008. Prolyl isomerase, Pin1: new findings of post-translational modifications and physiological substrates in cancer, asthma and Alzheimer's disease. Cell Mol Life Sci. 65:359-375.

70. Tatro, E.T., T.B. Nguyen, C.A. Bousman, E. Masliah, I. Grant, J.H. Atkinson, and I.P. Everall. 2010. Correlation of major depressive disorder symptoms with FKBP5 but not FKBP4 expression in human immunodeficiency virus-infected individuals. J Neurovirol. 16:399-404.

71. Thompson, P.M., K.M. Hayashi, G.I. De Zubicaray, A.L. Janke, S.E. Rose, J. Semple, M.S. Hong, D.H. Herman, D. Gravano, D.M. Doddrell, and A.W. Toga. 2004. Mapping hippocampal and ventricular change in Alzheimer disease. Neuroimage. 22:1754-1766.

72. Tsechpenakis, G., and S.P. Chatzis. 2011. Deformable probability maps: Probabilistic shape and appearance-based object segmentation. Computer Vision and Image Understanding. 115:1157-1169.

73. Wagner, K.V., D. Marinescu, J. Hartmann, X.D. Wang, C. Labermaier, S.H. Scharf, C. Liebl, M. Uhr, F. Holsboer, M.B. Muller, and M.V. Schmidt. 2012. Differences in FKBP51 regulation following chronic social defeat stress correlate with individual stress sensitivity: influence of paroxetine treatment. Neuropsychopharmacology. 37:2797-2808. 
74. Wang, C., M. Shen, B. Guillaume, Y.S. Chong, H. Chen, M.V. Fortier, M.J. Meaney, and A. Qiu. 2018. FKBP5 Moderates the Association between Antenatal Maternal Depressive Symptoms and Neonatal Brain Morphology. Neuropsychopharmacology. 43:564-570.

75. Wang, Y., B. Qiu, J. Liu, W.G. Zhu, and S. Zhu. 2014. Cocaine- and amphetamine-regulated transcript facilitates the neurite outgrowth in cortical neurons after oxygen and glucose deprivation through PTN-dependent pathway. Neuroscience. 277:103-110.

76. Williams, J.A., H.M. Ni, Y. Ding, and W.X. Ding. 2015. Parkin regulates mitophagy and mitochondrial function to protect against alcohol-induced liver injury and steatosis in mice. Am J Physiol Gastrointest Liver Physiol. 309:G324-340.

77. Wilson, C., and C. Gonzalez-Billault. 2015. Regulation of cytoskeletal dynamics by redox signaling and oxidative stress: implications for neuronal development and trafficking. Front Cell Neurosci. 9:381.

78. Xie, P., H.R. Kranzler, J. Poling, M.B. Stein, R.F. Anton, L.A. Farrer, and J. Gelernter. 2010. Interaction of FKBP5 with childhood adversity on risk for post-traumatic stress disorder. Neuropsychopharmacology. 35:1684-1692.

79. Xing, Y., J. Hou, Q. Meng, M. Yang, H. Kurihara, and J. Tian. 2015. Novel antidepressant candidate R0-05 modulated glucocorticoid receptors activation and FKBP5 expression in chronic mild stress model in rats. Neuroscience. 290:255-265.

80. Xu, K., G. Zhong, and X. Zhuang. 2013. Actin, spectrin, and associated proteins form a periodic cytoskeletal structure in axons. Science. 339:452-456.

81. Yadaw, A.S., M.M. Siddiq, V. Rabinovich, R. Tolentino, J. Hansen, and R. lyengar. 2019. Dynamic balance between vesicle transport and microtubule growth enables neurite outgrowth. PLoS Comput Biol. 15:e1006877.

82. Yang, F., Q. Jiang, J. Zhao, Y. Ren, M.D. Sutton, and J. Feng. 2005. Parkin stabilizes microtubules through strong binding mediated by three independent domains. J Biol Chem. 280:17154-17162.

83. Yogev, S., R. Cooper, R. Fetter, M. Horowitz, and K. Shen. 2016. Microtubule Organization Determines Axonal Transport Dynamics. Neuron. 92:449-460.

84. Yong, W., Z. Yang, S. Periyasamy, H. Chen, S. Yucel, W. Li, L.Y. Lin, I.M. Wolf, M.J. Cohn, L.S. Baskin, E.R. Sanchez, and W. Shou. 2007. Essential role for Co-chaperone Fkbp52 but not Fkbp51 in androgen receptor-mediated signaling and physiology. J Biol Chem. 282:5026-5036.

85. Yun, J.Y., M.J. Jin, S. Kim, and S.H. Lee. 2020. Stress-related cognitive style is related to volumetric change of the hippocampus and FK506 binding protein 5 polymorphism in post-traumatic stress disorder. Psychol Med:1-12.

86. Zannas, A.S., M. Jia, K. Hafner, J. Baumert, T. Wiechmann, J.C. Pape, J. Arloth, M. Kodel, S. Martinelli, M. Roitman, S. Roh, A. Haehle, R.T. Emeny, S. lurato, T. Carrillo-Roa, J. Lahti, K. Raikkonen, J.G. Eriksson, A.J. Drake, M. Waldenberger, S. Wahl, S. Kunze, S. Lucae, B. Bradley, C. Gieger, F. Hausch, A.K. Smith, K.J. Ressler, B. Muller-Myhsok, K.H. Ladwig, T. Rein, N.C. Gassen, and E.B. Binder. 2019. 
Epigenetic upregulation of FKBP5 by aging and stress contributes to NF-kappaB-driven inflammation and cardiovascular risk. Proc Natl Acad Sci U S A. 116:11370-11379.

87. Zhang, T., C. Hou, S. Zhang, S. Liu, Z. Li, and J. Gao. 2019. Lgl1 deficiency disrupts hippocampal development and impairs cognitive performance in mice. Genes Brain Behav. 18:e12605.

88. Zuiderveld, K. 1994. Contrast Limited Adaptive Histogram Equalization. . In Graphic Gems IV, Ed: P.S. Heckbert, Academic Press.

\section{Figures}

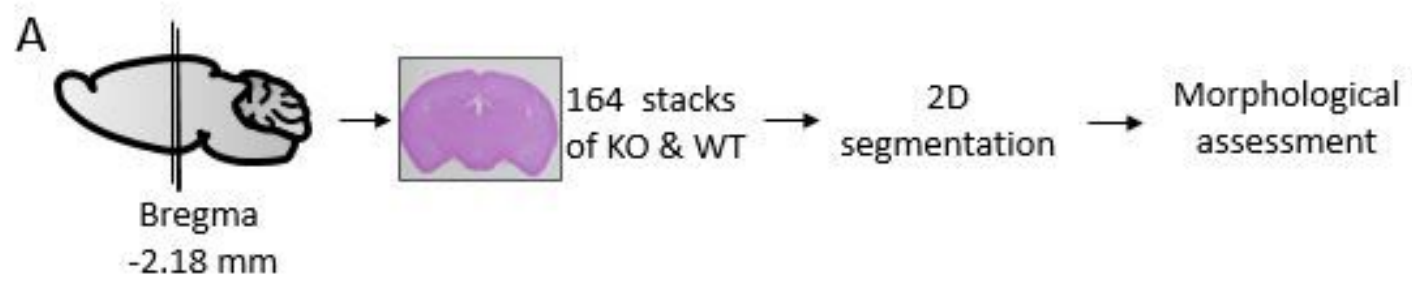

B
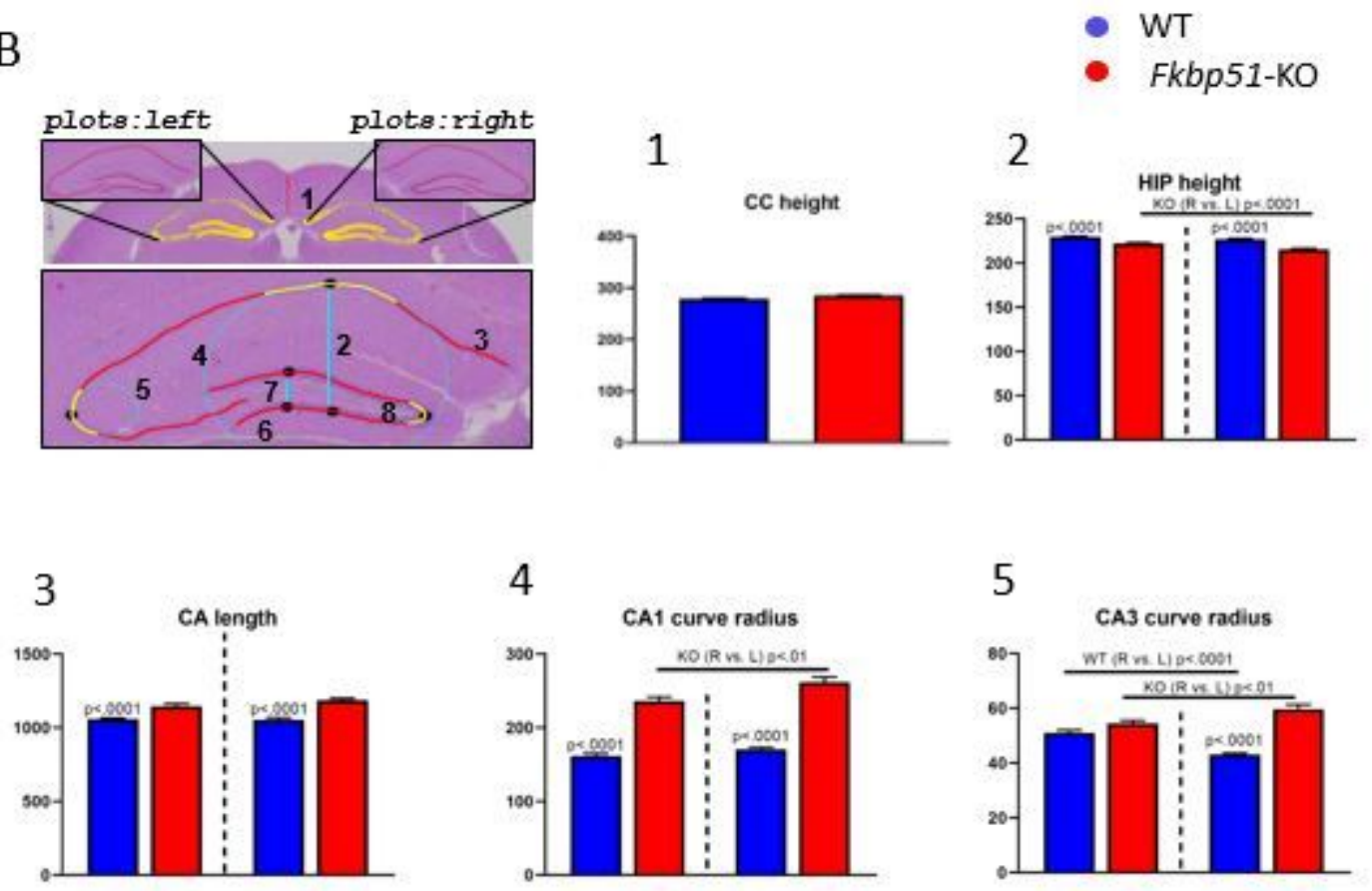

4

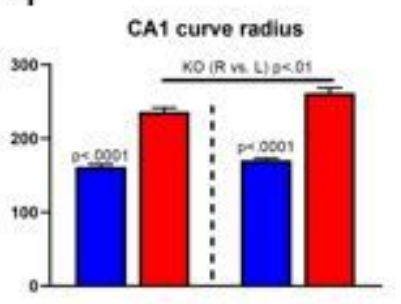

7

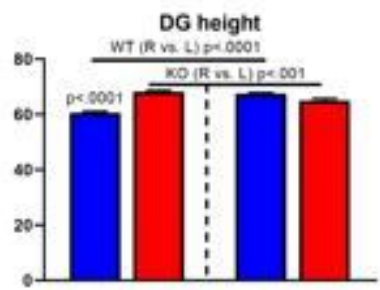

5

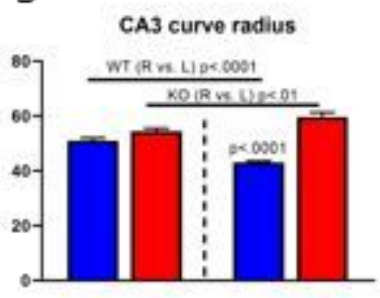

8

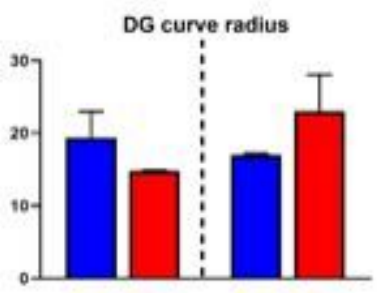




\section{Figure 1}

Ablation of the Fkbp51 gene compromises hippocampal size. (A) Image acquisition and data processing. Sagittal view with vertical lines indicating the position range where coronal sections were acquired. Stacks of images were analyzed from both WT and KO followed by 2D segmentation of areas of interest. Machine learning was applied and landmarks of CA and DG were analyzed. (B) Representative brain section with hippocampal subregions highlighted. The yellow regions indicate the $2 \mathrm{D}$ segmentation outcome, and red lines are the resulting smooth centerlines along the interface. Eight landmarks were measured, annotated in the magnified view at the bottom of the section. (1) the cortex plus corpus callosum (CC) length was approximated using the vertical intensity profile of the contrast enhanced grayscale image; (2) the hippocampus height was calculated in the vertical direction, after the "peak point" between CA1 and CA2 was located; (3) the length of the hippocampus was calculated as the arc length of the pyramidal cell layer; (4) the local curve change at the "peak point" between CA1 and CA2 was captured by the radius of the best locally fit circle to the curve (in cyan); (5) similarly, the local curve change of CA3 was captured by the radius of the best locally fit circle; (6) the length of the colored DG segment was calculated as the arc lengths of the corresponding granular cell layer; (7) the DG height was measured vertically, after locating the peak point between the top and bottom granular cell layers; (8) the DG apex was captured by the radius of the best locally fit circle to the DG line. The y-axis in each plot corresponds to measurement in pixels, and the scale varies across the plots for illustration purposes. In blue and red are the measurements for WT and KO, respectively. The plots 2-8 are divided by a black vertical line into left and right hippocampus segment measurements. Bold dots indicate the group averages. CA: cornu ammonis. DG: dentate gyrus; Hippo: hippocampus. 


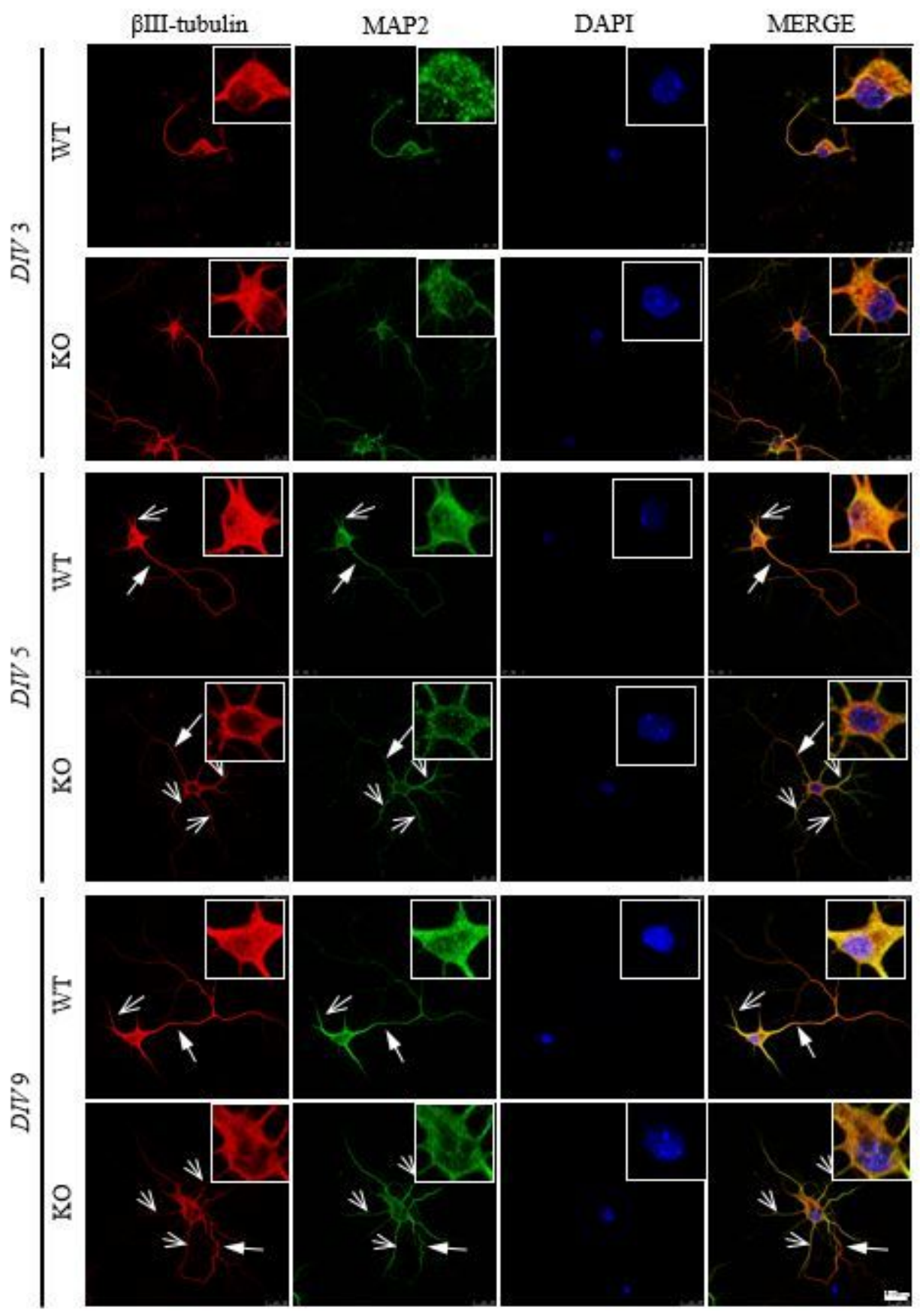

\section{Figure 2}

Morphological differences between Fkbp51 KO and WT primary cultured neurons Neurons were labeled with $\beta$ III-tubulin for whole neurons (red), MAP2 for soma and dendrites (green), and DAPI for nuclei (blue). Primary cultured neurons were analyzed at DIV3, DIV5, and DIV9. Solid head arrows indicate axon outgrowth, while line head arrows indicate dendrite outgrowth. Inset of each image showed the magnified staining of soma area. Scale bar $=100 \mu \mathrm{m}$. DIV: days in vitro. Immunofluorescence labeling of $\beta$ IIItubulin, F-actin, and Tau is included in the Supplementary Information, Figure S2. 
A

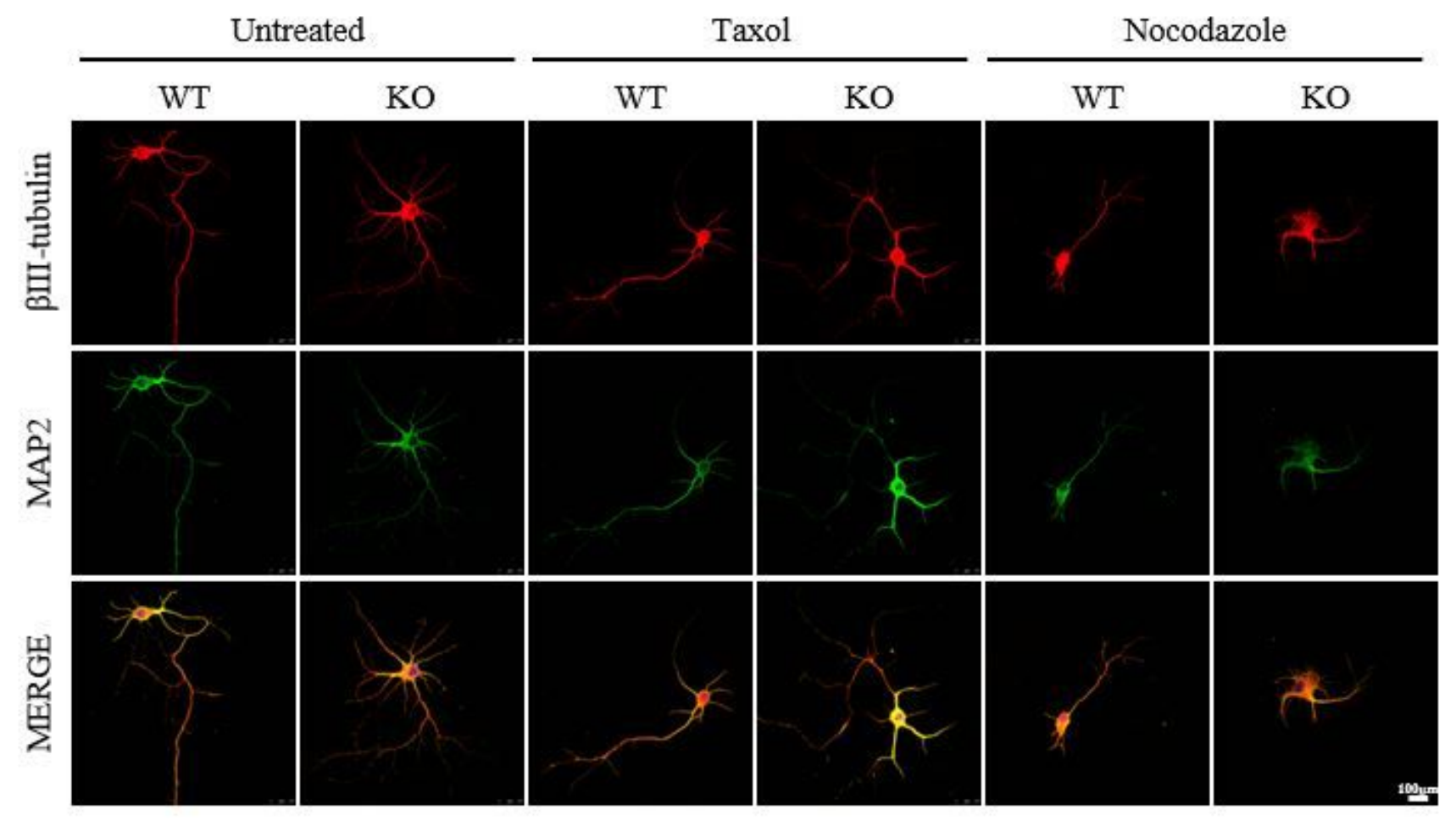

B

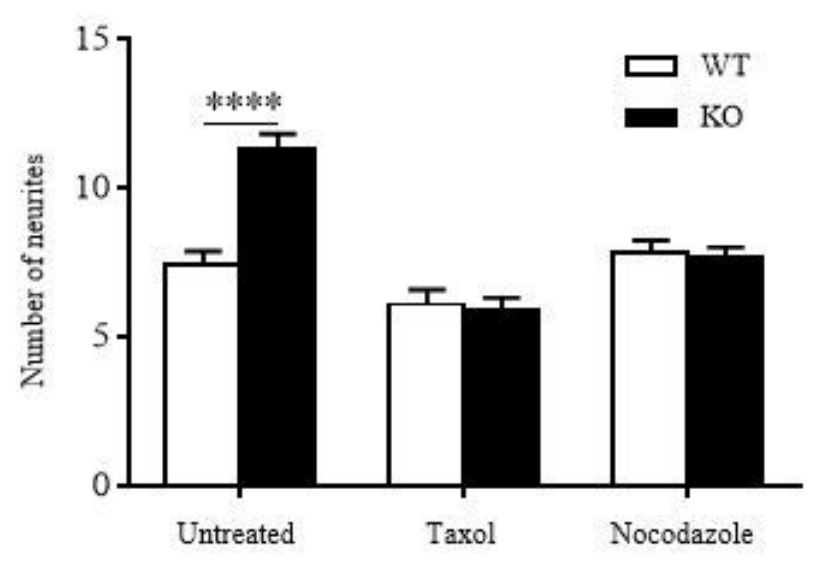

Figure 3

Fkbp51 KO neuronal polarization sensitive to microtubule stability alteration (A) Morphological differences between Fkbp51 KO and WT primary cultured neurons are observable at DIV 9. Neurons are labeled with $\beta$ III-tubulin (red) and MAP2 (green). Hippocampal neurons from Fkbp51 KO mice exhibit enhanced neurite outgrowth. Taxol reduced dendrite outgrowth from Fkbp51 KO neurons, while nocodazole reduced minor neurites formation in both WT and Fkbp51 KO, with more obvious neuronal 
deformation present in Fkbp51 KO. Scale bar $=100 \mu \mathrm{m}$. (B) Statistical analysis of neurite numbers in

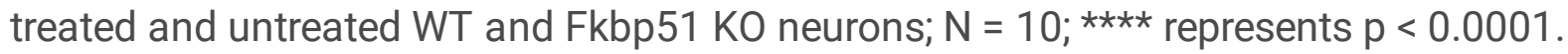

Figure. 4
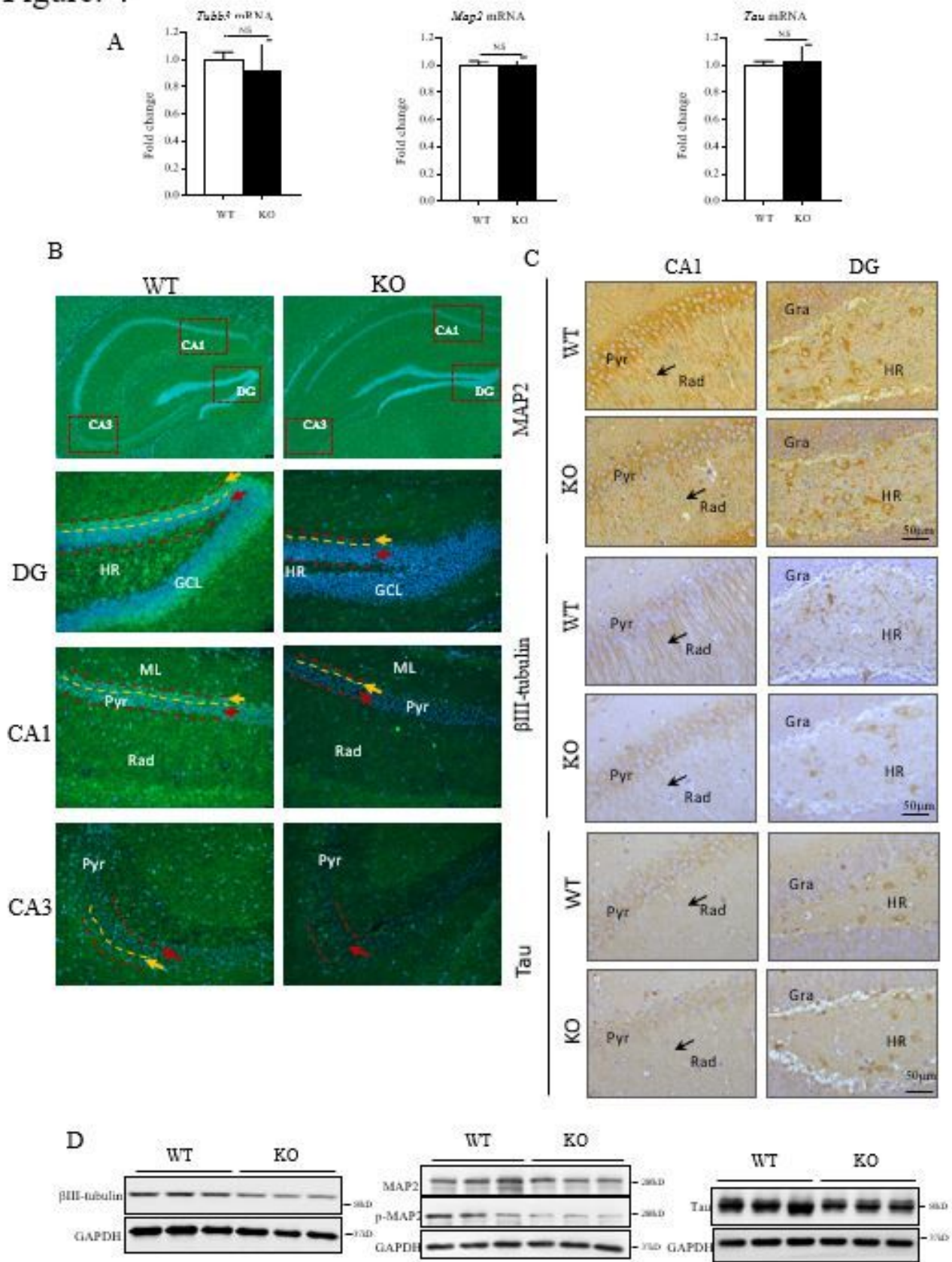

$E$
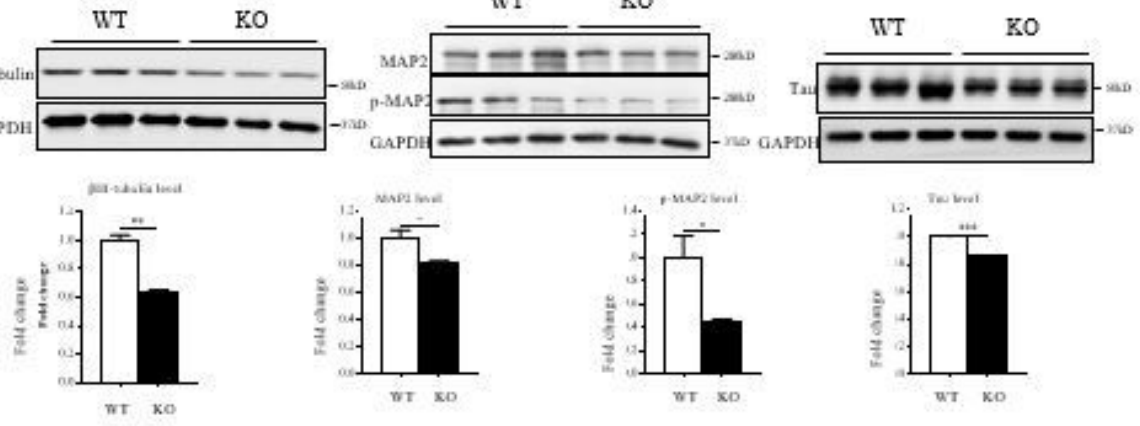

\section{Figure 4}

In vivo differences in Blll-tubulin, MAP2, and Tau expression in the hippocampus (A) No significant differences were found in the expression levels of Tubb3, Map2, or Tau mRNA. (B) MAP2 and DAPI immunofluorescent labeling of hippocampus and magnified subfields including DG, CA1, and CA3. (C) (A- 
top panel) In the CA1 and DG hippocampal subfields, MAP2 and BIII-tubulin expression are significantly lower in the Fkbp51 KO than in the WT, particularly in the Rad. Tau protein expression levels are also lower in the Fkbp51 KO than in the WT (scale bar $=50 \mu \mathrm{m}$ ). (D) Western blotting confirmed the reduced expression of the MAP2, p-MAP2, ßlll-tubulin, and Tau proteins. (E) Quantification of the MAP2, p-MAP2, $\beta$ III-tubulin and Tau proteins in the hippocampus. Data represent the mean \pm SEM normalized to GAPDH. * represents $p<0.05$ and ** represents $p<0.01$. Pyr: pyramidal cell layer; Rad: radiatum layer; HR: hilar region; GCL: granule cell layer.
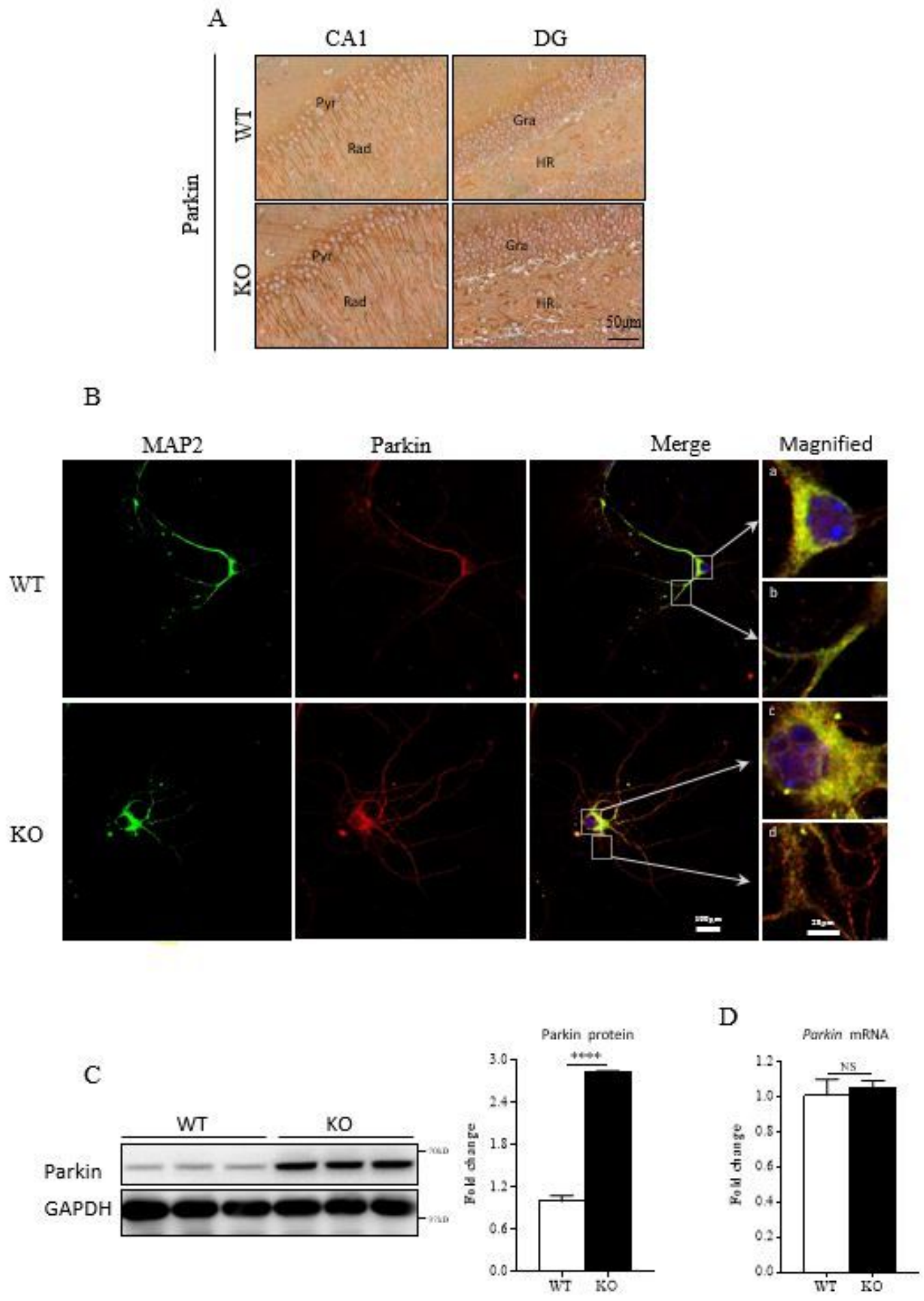


\section{Figure 5}

Increased Parkin levels occur in Fkbp51 KO mice via post-translational regulation. (A) Parkin expression is significantly increased in the Fkbp51 KO in the hippocampal subregions CA1 and DG, scale bar $=50$ $\mu \mathrm{m}$. (B) Primary cultured WT and Fkbp51 KO hippocampal neurons labeled at DIV9 with MAP2 (green), Parkin (red), and DAPI (blue), scale bar $=100 \mu \mathrm{m}$. WT neurons express more MAP2 but less Parkin than Fkbp51 KO neurons in the soma and dendrites. The magnified panel highlights the distinct labeling patterns in WT and Fkbp51 KO soma and dendrites. (C) Western blotting confirms significantly higher Parkin protein expression in the Fkbp51 KO hippocampus. Quantification of the Western blot density indicates that the Parkin protein is significantly elevated in the Fkbp51 KO hippocampus (over 2.5-fold). (D) No significant differences were found in Parkin mRNA expression. Data represent the mean \pm SEM normalized to GAPDH. ${ }^{\star \star \star}$ represents $p<0.001$. Pyr: pyramidal cell layer; Rad: radiatum layer; HR: hilar region; GCL: granule cell layer. 


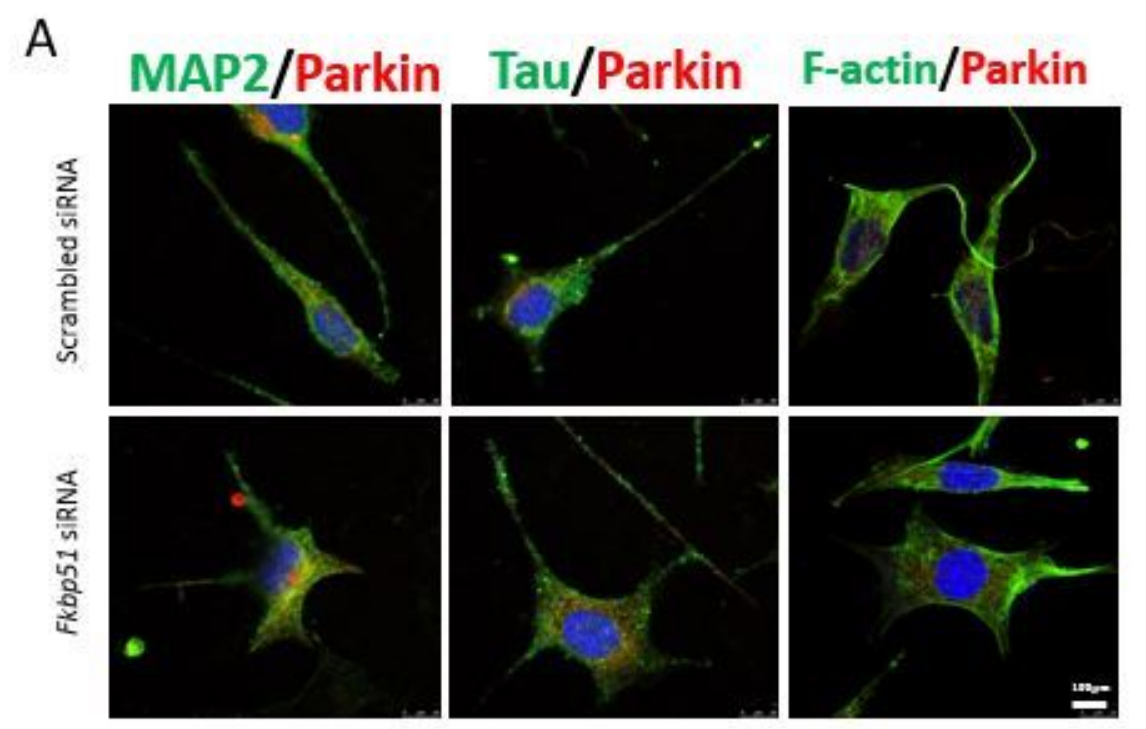

B

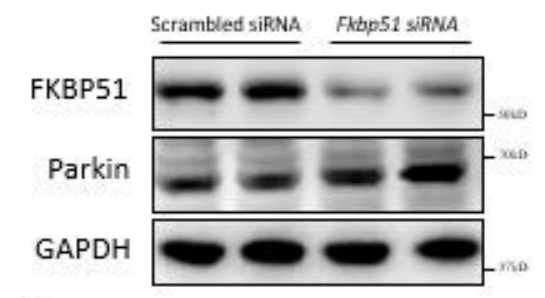

D

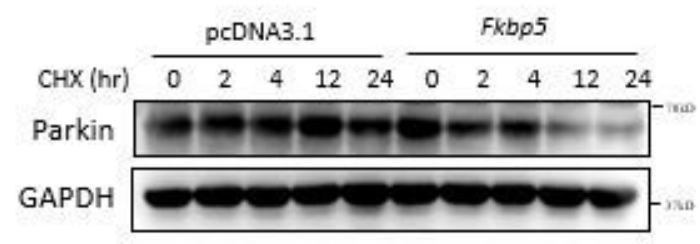

C

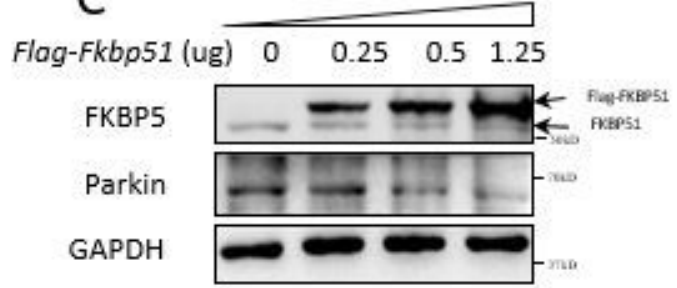

E

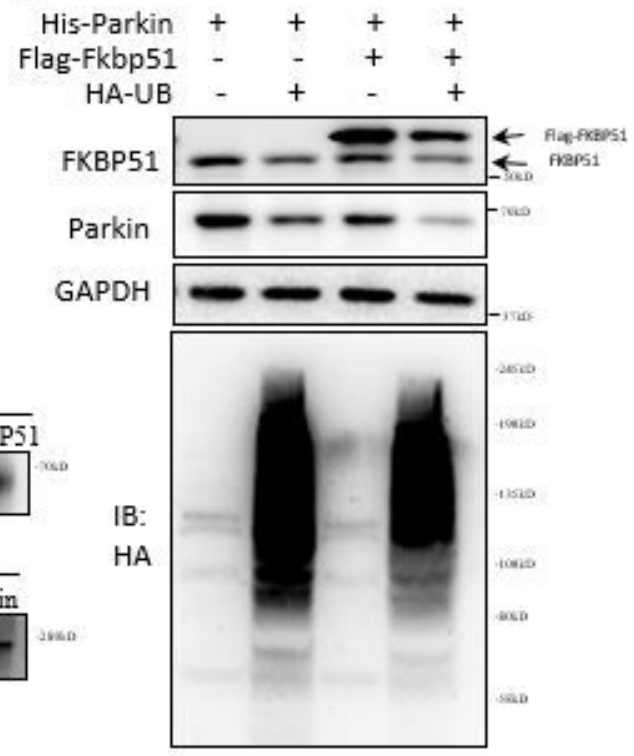

\section{Figure 6}

FKBP51 prevents disrupted SH-SY5Y cell morphology and is associated with ubiquitin-mediated degradation of Parkin. (A) Double labeling of MAP2/Parkin, Tau/Parkin, and F-actin/Parkin indicates that Fkbp51 siRNA treatment increases the outgrowth of neurites in SH-SY5Y cells, decreases MAP2, Tau, and F-actin expression, and increases Parkin expression. (B) Western blotting confirms reduced FKBP51 and increased Parkin levels following Fkbp51 siRNA treatment compared to those of the scramble siRNA 
control. (C) Increased expression of Flag-FKBP51 in SH-5YSY cells corresponds to a dose-dependent decrease in Parkin protein levels. (D) Parkin expression levels decreased with overexpression of Fkbp51 but not pcDNA3.1 control. (E) Co-transfection of His-Parkin with ubiquitin HA-UB alone (lane 2), FlagFKBP51 and His-Park (lane 3), or HA-UB and Flag-FKBP51 together (lane 4) promotes ubiquitin-dependent degradation of both FKBP51 and Parkin. (F) Co-IP assays show that FKBP51 binds Parkin, Blll-tubulin, and Tau. Parkin also binds MAP2.

\section{WT}
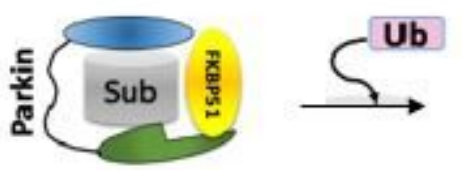

Fkbp51 Inhibition

Reduces Parkin activity

Reduces substrate degradation

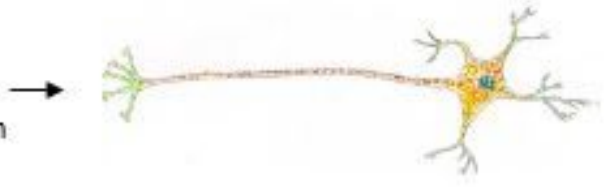

$\mathrm{KO}$
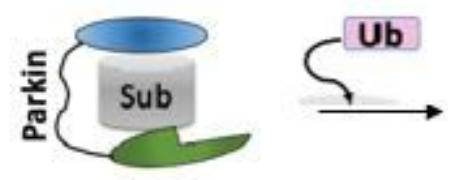

Lack of Fkbp51 inhibition

Enhances Parkin activity

Enhances substrate degradation

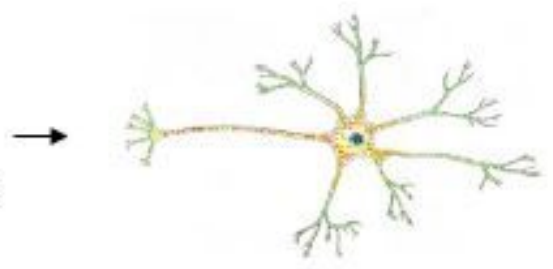

\section{Figure 7}

Model of morphological and molecular alterations in WT and Fkbp51 KO neuron polarization. A schematic model demonstrating the morphological differences between WT and Fkbp51 KO neurons, with shorter axons and greater dendritic outgrowth in Fkbp51 KO neurons. The underlying mechanism could be due to Fkbp51 inhibition of Pakin. In WT neurons, FKBP51 interacts with Parkin. The interaction reduced the Parkin activity. The loss of FKBP51 affects the Parkin-FKBP51 interaction, resulting in enhanced Parkin accumulation and enhanced Parkin activity.

\section{Supplementary Files}

This is a list of supplementary files associated with this preprint. Click to download.

- Supplementaryinformation050521.docx

- SupplementaryTable1.docx

- ga.jpg

- S1.jpg

- S2.jpg

- S3.jpg 